\title{
Hyper-X Post-Flight Trajectory Reconstruction
}

\author{
Christopher D. Karlgaard* \\ Analytical Mechanics Associates, Inc., Hampton, VA 23666 \\ Paul V. Tartabini ${ }^{\dagger}$ \\ NASA Langley Research Center, Hampton, VA 23681 \\ Robert C. Blanchard ${ }^{\ddagger}$ and Michael Kirsch ${ }^{\S}$ \\ The George Washington University, Hampton, VA 23666 \\ Matthew D. Toniolo \\ Analytical Mechanics Associates, Inc., Hampton, VA 23666
}

\begin{abstract}
This paper discusses the formulation and development of a trajectory reconstruction tool for the NASA $\mathrm{X}-43 \mathrm{~A} /$ Hyper- $\mathrm{X}$ high speed research vehicle, and its implementation for the reconstruction and analysis of flight test data. Extended Kalman filtering techniques are employed to reconstruct the trajectory of the vehicle, based upon numerical integration of inertial measurement data along with redundant measurements of the vehicle state. The equations of motion are formulated in order to include the effects of several systematic error sources, whose values may also be estimated by the filtering routines. Additionally, smoothing algorithms have been implemented in which the final value of the state (or an augmented state that includes other systematic error parameters to be estimated) and covariance are propagated back to the initial time to generate the best-estimated trajectory, based upon all available data. The methods are applied to the problem of reconstructing the trajectory of the Hyper-X vehicle from flight data.
\end{abstract}

\section{Introduction}

$\mathrm{T}$ NASA Hyper-X Program is a joint Langley Research Center and Dryden Flight Research Center 1 effort to develop a hypersonic airbreathing research vehicle, powered by an airframe-integrated supersonic combustion ramjet (scramjet) propulsion system. Major objectives of the program are to acquire data on the operation of the scramjet propulsion system and to demonstrate controlled hypersonic flight technologies. ${ }^{1,2}$ A secondary goal is to evaluate the experimental ${ }^{3-6}$ and computational ${ }^{7-10}$ techniques used in the design and analysis of the Hyper-X vehicle in order to facilitate future hypersonic and launch vehicle programs. ${ }^{1,11,12}$

The flight experiment mission profile is shown in Fig. 1, and takes place in the Western Test Range over the Pacific Ocean. The experiment begins with a boost phase, where the Hyper-X Launch Vehicle (HXLV) stack (shown in Fig. 2), consisting of the X-43A/Hyper-X Research Vehicle (HXRV), shown in Fig. 3, mated to a modified Pegasus booster, is dropped from the NASA B-52 aircraft at an altitude of approximately 40,000 ft and ascends to an altitude of approximately 100,000 ft. At this point, the HXRV separates from the stack, stabilizes, and the scramjet propulsion experiment begins, lasting several seconds. Following the engine test, the vehicle follows a descent trajectory that includes various maneuvers that are optimized for the post-flight extraction of stability and control derivatives and other aerodynamic properties (e.g., Ref. 13). It is important to point out that approximately one minute prior to the drop time, the on-board navigation

\footnotetext{
*Project Engineer, Member AIAA.

${ }^{\dagger}$ Aerospace Engineer, Member AIAA.

$\ddagger$ Professor, Joint Institute for the Advancement of Flight Sciences, Associate Fellow AIAA.

$\S$ Graduate Student, Joint Institute for the Advancement of Flight Sciences, Student Member AIAA.

ฯProject Engineer
} 


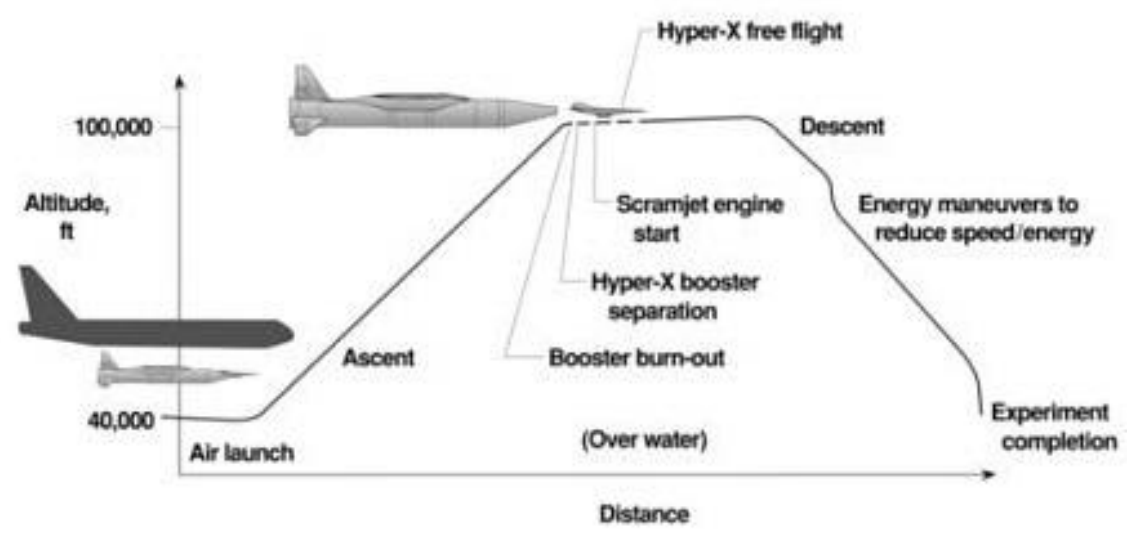

Figure 1. Hyper-X Flight Trajectory

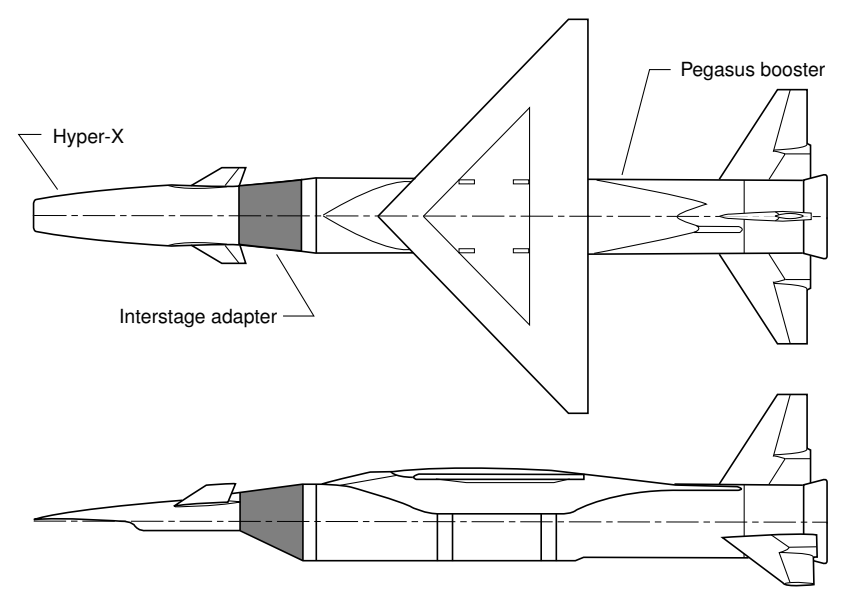

Figure 2. Hyper-X Launch Vehicle Stack Configuration

system switches from a satellite-aided system to a free inertial system. Therefore, drift is expected in the on-board navigation solution after this time.

A critical component of the post-flight data reduction and analysis tasks to determine propulsion system performance and the extraction of various aerodynamic coefficients is the reconstruction of the vehicle trajectory. The trajectory reconstruction process uses in-flight telemetry data combined with a variety of redundant measurements to generate maximum-likelihood estimates of the vehicle position, velocity, and orientation time histories, along with estimates of the uncertainty associated with them. The Hyper-X vehicle has many instruments that gather data to be used for this process, such as the Inertial Measurement Unit (IMU), Global Positioning System (GPS), and Flush Air Data Systems (FADS). Numerous other useful observations and measurements are available from ground-based radar tracking stations and from instruments carried on board the B-52 carrier vehicle.

A tool known as the Statistical Trajectory Estimation Program (STEP) $)^{14-16}$ has been developed for post-flight trajectory reconstruction and parameter identification in support of maneuvering re-entry vehicle flight projects in the 1960's. STEP uses a recursive, minimum variance filtering algorithm to process accelerometer and gyroscope measurements recorded by the IMU during the flight, as well as position and velocity measurements from outside sources, such as ground based radar tracking stations, to determine a maximum-likelihood trajectory and user defined systematic error sources along with an estimate of the accuracy of these parameters. STEP allows the user to specify which systematic error sources should be estimated, such as an accelerometer bias, and is general with the type of outside tracking measurements available. STEP and its variants have been applied to a variety of post-flight planetary entry trajectory reconstruction and analysis problems, particularly the SV-5D/X-23A maneuverable entry vehicle, ${ }^{17}$ the Viking 


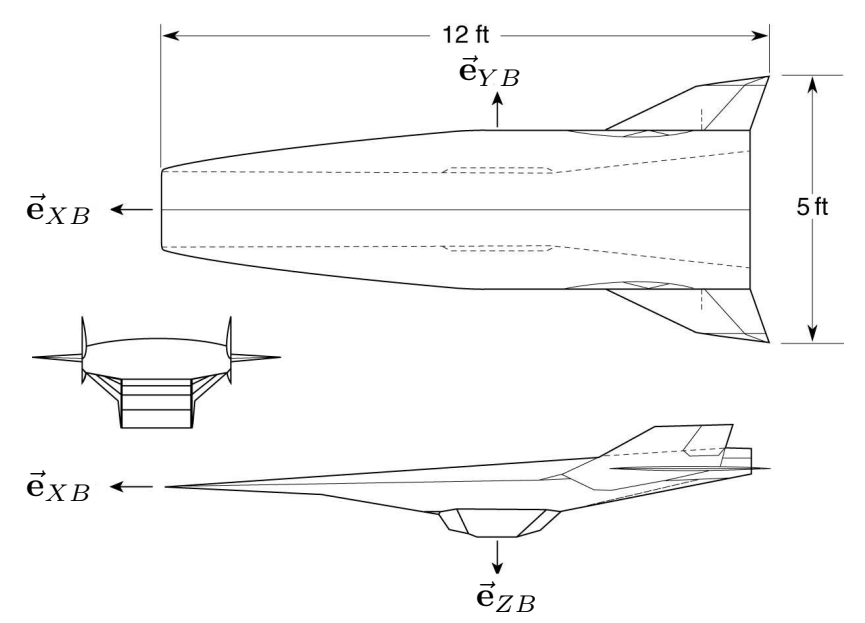

Figure 3. Hyper-X Research Vehicle Configuration

landers, ${ }^{18-20}$ Pioneer, ${ }^{21}$ and the Space Shuttle, ${ }^{22,23}$ among others.

STEP is not without drawbacks, however. The STEP user interface is poor, and the source code is archaic and not commented. The latter issue makes the STEP algorithm difficult to check or modify to suit algorithmic developments or new types of data sources. With these limitations in mind, an effort was made to assess how STEP may be modernized both in terms of its underlying filter algorithm and its implementation. ${ }^{24}$ This paper discusses the application of several nonlinear filtering algorithms to the post-flight trajectory reconstruction problem, wherein the inertial accelerations and angular rates measured by the IMU are used to integrate the nonlinear equations of motion, while GPS observations of position and velocity are used to provide corrections to the trajectory estimate. These corrections are incorporated into the state estimate at the measurement points through use of an Extended Kalman Filter (EKF) ${ }^{25-28}$ for a forward and backward pass through the data. The results of the two filtering passes are combined using the Fraser-Potter smoothing method ${ }^{26,29}$ to determine the final trajectory estimate as a function of time.

Similar methods have been recently applied to the entry trajectory estimation problem in Refs. 30-32 using a variety of Kalman filtering and smoothing techniques. Unfortunately, these methods do not model the vehicle rotational dynamics, and furthermore, Refs. 31 and 32 are not formulated for cases where the vehicle measures its acceleration and angular rates. Therefore, the methods of Refs. 30-32 are not directly applicable to the problem investigated in this paper. Other proposed techniques for post-flight trajectory reconstruction simply use either a numerical integration of the raw IMU acceleration and angular rate data, or the on-board navigation solution, to produce estimates of position, velocity, and orientation, ${ }^{33,34}$ but do not make use of all available observation data through the application of a filtering and smoothing process to correct for various drifts, as is discussed in the next section.

\section{Filtering Equations}

The current section shall be concerned with the problem of estimating the state of a nonlinear system of ordinary differential equations

$$
\dot{\mathbf{x}}(t)=\mathbf{f}(\mathbf{x}(t), \mathbf{v}(t), t)
$$

where $\mathbf{x}$ is the matrix of the system states, $\mathbf{f}(\mathbf{x}, \mathbf{v}, t)$ is a nonlinear function that describes the evolution of the system state, and $\mathbf{v}$ is a random process with covariance matrix $\mathbf{Q}$ given by $\mathbf{Q}=E\left(\mathbf{v v}^{T}\right)$ and mean $\overline{\mathbf{v}}=E(\mathbf{v})$, where $E$ is the expectation of the random variable. The state estimation procedure requires knowledge of independent observations of some quantity which may be related to the value of the state by the nonlinear algebraic equations

$$
\mathbf{y}(t)=\mathbf{g}(\mathbf{x}(t), \mathbf{w}(t), t)
$$

given at discrete time intervals, where $\mathbf{y}$ is the value of the observation, $\mathbf{g}$ is the nonlinear relationship between the states and the observations, and $\mathbf{w}$ is a random measurement noise variable with covariance matrix $\mathbf{R}$ and mean $\overline{\mathbf{w}}$. 


\section{A. The Extended Kalman Filter}

The extended Kalman filter (EKF) is a somewhat ad-hoc modification of the linear Kalman filter in which the linear state transition is replaced by a numerical integration of the full nonlinear dynamic model in between measurement updates, before applying the Kalman update at each measurement time. In addition, the nonlinear relationship between the state and measurement data is used to calculate the residuals rather than the linear approximate relationship. The EKF uses the current estimated state as the reference conditions for computing the system dynamics matrices required to calculate the Kalman gain matrix at each time step. The algorithm is given by ${ }^{25-28}$

$$
\hat{\mathbf{x}}_{k}=\overline{\mathbf{x}}_{k}+\mathbf{K}_{k}\left[\mathbf{y}_{k}-\mathbf{g}\left(\overline{\mathbf{x}}_{k}, \overline{\mathbf{w}}_{k}, t_{k}\right)\right]
$$

where $\overline{\mathbf{x}}_{k}$ is the predicted value of the state at time increment $k, \mathbf{y}_{k}$ is the value of the measurement data, and $\mathbf{g}\left(\overline{\mathbf{x}}_{k}, \overline{\mathbf{w}}_{k}, t_{k}\right)$ is the predicted value of the measurement data at time $t_{k}$, following from the propagation of the state estimate from time $t_{k-1}$ to time $t_{k}$. The gain and covariance equations are given by

$$
\begin{aligned}
\mathbf{K}_{k} & =\overline{\mathbf{P}}_{k} \mathbf{C}_{k}^{T}\left(\mathbf{C}_{k} \overline{\mathbf{P}}_{k} \mathbf{C}_{k}^{T}+\mathbf{D}_{k} \mathbf{R}_{k} \mathbf{D}_{k}^{T}\right)^{-1} \\
\hat{\mathbf{P}}_{k} & =\left(\mathbf{I}-\mathbf{K}_{k} \mathbf{C}_{k}\right) \overline{\mathbf{P}}_{k}
\end{aligned}
$$

where $\overline{\mathbf{P}}_{k}$ is the solution of the matrix differential equation

$$
\begin{aligned}
\dot{\overline{\mathbf{P}}}(t) & =\mathbf{A} \overline{\mathbf{P}}+\overline{\mathbf{P}}^{T} \mathbf{A}^{T}+\mathbf{B Q B}^{T} \\
\overline{\mathbf{P}}\left(t_{k-1}\right) & =\hat{\mathbf{P}}_{k-1}
\end{aligned}
$$

at time $t_{k}$, calculated through numerical integration. The matrices $\mathbf{A}, \mathbf{B}, \mathbf{C}$ and $\mathbf{D}$ used in the Kalman gain calculations are found by linearizing the system dynamics about the current state estimate, $\hat{\mathbf{x}}_{k}$, so that

$$
\begin{aligned}
& \mathbf{A}(t)=\left.\frac{\partial \mathbf{f}}{\partial \mathbf{x}}\right|_{\mathbf{x}=\hat{\mathbf{x}}_{k}, \mathbf{v}=\mathbf{0}, t} \\
& \mathbf{B}(t)=\left.\frac{\partial \mathbf{f}}{\partial \mathbf{v}}\right|_{\mathbf{x}=\hat{\mathbf{x}}_{k}, \mathbf{v}=\mathbf{0}, t} \\
& \mathbf{C}(t)=\left.\frac{\partial \mathbf{g}}{\partial \mathbf{x}}\right|_{\mathbf{x}=\hat{\mathbf{x}}_{k}, \mathbf{w}=\mathbf{0}, t} \\
& \mathbf{D}(t)=\left.\frac{\partial \mathbf{g}}{\partial \mathbf{w}}\right|_{\mathbf{x}=\hat{\mathbf{x}}_{k}, \mathbf{w}=\mathbf{0}, t}
\end{aligned}
$$

The EKF formulation is superior to that implemented in the original STEP code in Refs. 14-16. The STEP implementation made use of linearized equations of motion for state propagation between measurement points, as well as linearized representations of the measurement equations for computing the residuals. This linearization was performed about the current estimate, as is used by the EKF for gain calculations, or about a pre-defined nominal trajectory; the latter technique is better known today as a neighboring-optimal Kalman filter ${ }^{27}$ or as a linearized Kalman filter. ${ }^{28}$

Note that in the current formulation of the filtering equations, the state $\mathbf{x}$ could include both dynamic states as well as constant model parameters to be estimated. Likewise, the input noise $\mathbf{v}$ could include random inputs to reflect the model uncertainty (process noise), or the uncertainty associated with model parameters that are not included in the state $\mathbf{x}$. The uncertain parameters from the latter case are commonly known as "consider parameters." The two noise inputs to the system dynamics both seek to degrade the state covariance matrix in order to reflect uncertainty in the model, but are different in the sense that the process noise accounts for effects that are unknown whereas the consider parameters account for effects that are known but uncertain. A Kalman filter designed to include parametric uncertainty is typically known as a Kalman-Schmidt filter. ${ }^{25}$ It is worth noting that the STEP filter was designed to have the option of using a variety of consider parameters, but did not include a means of incorporating process noise into the estimation procedure.

The linearized Kalman-Schmidt filter algorithm implemented in STEP is computationally efficient and well understood. However, the algorithm has proven itself to be sensitive to the quality of the nominal trajectory or the estimated trajectory, depending on the mode of operation. If the reference trajectory is not an accurate representation of the true flight path, then the assumption of local linearity is violated and the 
filter often diverges, hence the algorithm must be modified to take nonlinearity into consideration, which is the advantage of the EKF method.

The EKF algorithm implemented in this paper may be used for both a forward pass and a backward pass through the measurement data. The final estimate from the forward pass through the data is used as the initial condition for the backward pass. This process can also be iterated globally using the change in the initial condition to measure the convergence of the estimates. The final trajectory is then computed as a linear combination of the forward and backward passes using the Fraser-Potter method, ${ }^{26,29}$ which is the subject of the next section.

\section{B. The Fraser-Potter Smoother}

The optimal smoothed solution is the blend of the forward and backward estimates of the state time history as output from the EKF algorithm. The Fraser-Potter method ${ }^{26,29}$ seeks to determine the smoothed solution at any instant in time as a linear combination of the forward and and backward filtered solution. The smoothed solution at a time $k$ is given by

$$
\begin{aligned}
\hat{\mathbf{P}}_{k} & =\left[\hat{\mathbf{P}}_{f_{k}}^{-1}+\hat{\mathbf{P}}_{b_{k}}^{-1}\right]^{-1} \\
\hat{\mathbf{x}}_{k} & =\hat{\mathbf{P}}_{k}\left[\hat{\mathbf{P}}_{f_{k}}^{-1} \hat{\mathbf{x}}_{f_{k}}+\hat{\mathbf{P}}_{b_{k}}^{-1} \hat{\mathbf{x}}_{b_{k}}\right]
\end{aligned}
$$

where $\hat{\mathbf{P}}$ is the smoothed covariance estimate, and $\hat{\mathbf{x}}$ is the smoothed state estimate, $\hat{\mathbf{P}}_{f}$ and $\hat{\mathbf{P}}_{b}$ are the covariance estimates from the forward and backward filter, respectively, and $\hat{\mathbf{x}}_{f}$ and $\hat{\mathbf{x}}_{b}$ are the state estimates from the forward and backward filter. By inspecting Eqs. (12-13) it is apparent that the smoother combines the forward estimate at time $t_{k}$, which makes use of all measurement data from the beginning of the data set to time $t_{k}$, with the backward estimate at time $t_{k}$, which makes use of all data from time $t_{k}$ to the end of the interval. In other words, the smoother makes use of all available data at each point of interest.

It should be noted that the original STEP code did not make use of an statistical smoothing method as is discussed in this section. STEP conducted smoothing by simply integrating the final state estimate back to the initial time via Eq. (1). The flaw with this approach is that it does not account for model uncertainty, and is also subject to numerical error buildup over large fit spans. These problems are eliminated by using the forward-backward filter implementation, combined together in the smoother.

\section{Dynamic Model}

The dynamic model discussed in this section is similar to that implemented in the original STEP. The equations of motion are based upon Newton's laws of motion, and are developed in a local, non-inertial coordinate system. Similar to the formulation used in STEP, the inertial acceleration and angular rate measurements recorded during the flight are used in place of detailed aerodynamic and propulsion force and moment models. An approximate representation of the complete gravitational potential is used to develop an expression for the gravitational force acting upon the vehicle. These assumptions lead to a system of nonlinear ordinary differential equations that describe the motion of the vehicle. The summary to follow begins with a definition of the required coordinate systems.

\section{A. Coordinate Systems}

This section provides a brief review of the coordinate systems used to develop the nonlinear equations of motion used in the trajectory reconstruction process. Firstly, the Earth Centered Inertial (ECI) frame is the frame fixed at the center of the Earth, or any other primary body of interest. The fundamental plane of this coordinate system is the equatorial plane of the Earth, with the $x$-axis, $\overrightarrow{\mathbf{e}}_{X I}$, aligned with the vernal equinox, the $z$-axis, $\overrightarrow{\mathbf{e}}_{Z I}$, in the direction of the planetary axis of rotation, and $y$-axis, $\overrightarrow{\mathbf{e}}_{Y I}$, chosen in such a way as to complete a right-handed coordinate system. This frame is obviously not truly inertial, although for short periods of time the inertial assumption is a valid and common approximation.

The Earth Centered Earth Fixed $(E C E F)$ frame is the coordinate system whose origin is located at the center of the Earth, similar to the ECI frame, but the coordinate axes are fixed with respect to the planet surface. The $x$-axis, $\overrightarrow{\mathbf{e}}_{X F}$, and the $z$-axis, $\overrightarrow{\mathbf{e}}_{Z F}$, are aligned with the prime meridian and the axis of planetary 


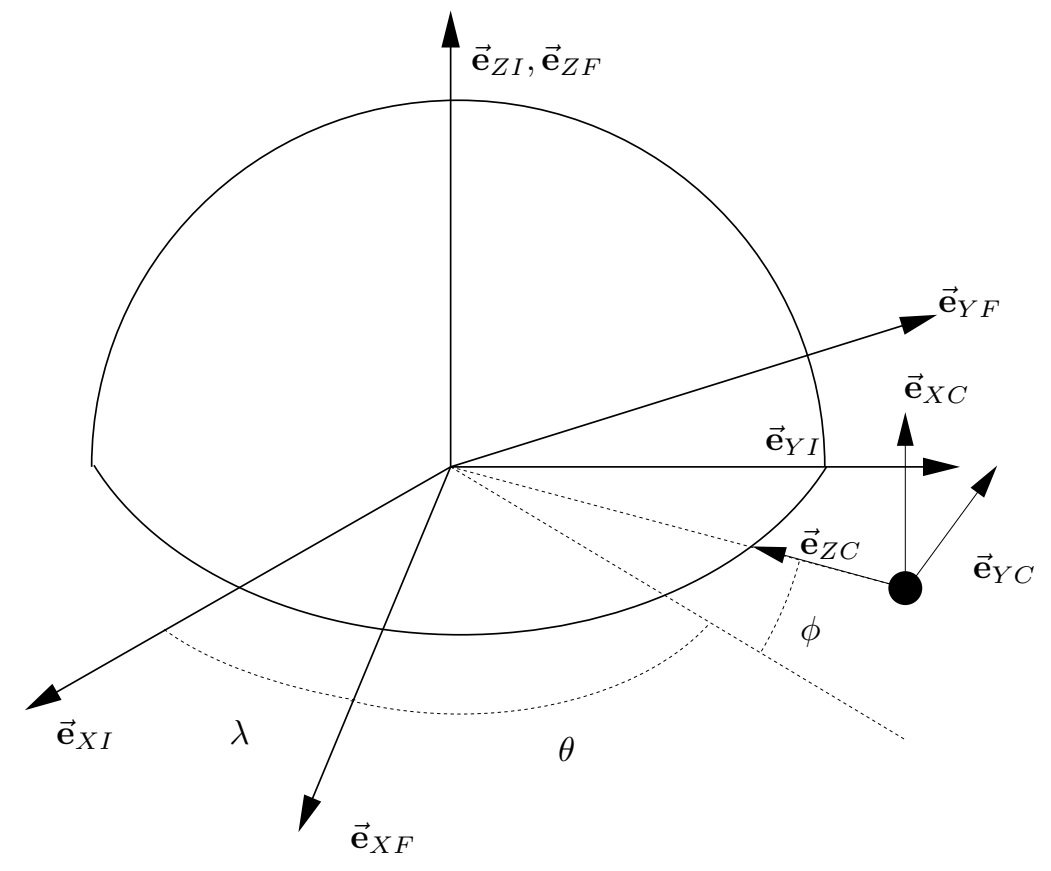

Figure 4. ECI, ECEF, and GC Frames

rotation, respectively. The $E C E F$ and $E C I$ frames may be related by the simple transformation

$$
\begin{aligned}
\left\{\begin{array}{c}
\overrightarrow{\mathbf{e}}_{X F} \\
\overrightarrow{\mathbf{e}}_{Y F} \\
\overrightarrow{\mathbf{e}}_{Z F}
\end{array}\right\} & =\mathbf{G}_{E C I 2 E C E F}\left\{\begin{array}{c}
\overrightarrow{\mathbf{e}}_{X I} \\
\overrightarrow{\mathbf{e}}_{Y I} \\
\overrightarrow{\mathbf{e}}_{Z I}
\end{array}\right\} \\
\mathbf{G}_{E C I 2 E C E F} & =\left[\begin{array}{ccc}
\cos \lambda & \sin \lambda & 0 \\
-\sin \lambda & \cos \lambda & 0 \\
0 & 0 & 1
\end{array}\right]
\end{aligned}
$$

where $\lambda$ is the sidereal angle of the prime meridian.

The geocentric $(G C)$ frame is one whose origin is located at the center of mass of the vehicle, and whose $z$-axis, $\overrightarrow{\mathbf{e}}_{Z C}$, is aligned in the direction of the origin of the $E C I$ (and $E C E F$ ) frame. The $x$-axis of the this frame, $\overrightarrow{\mathbf{e}}_{X C}$, is perpendicular to the $z$-axis, and points in the general direction of north. The $G C$ frame may be related to the $E C E F$ frame by the transformation

$$
\begin{aligned}
\left\{\begin{array}{c}
\overrightarrow{\mathbf{e}}_{X C} \\
\overrightarrow{\mathbf{e}}_{Y C} \\
\overrightarrow{\mathbf{e}}_{Z C}
\end{array}\right\} & =\mathbf{G}_{E C E F 2 G C}\left\{\begin{array}{c}
\overrightarrow{\mathbf{e}}_{X F} \\
\overrightarrow{\mathbf{e}}_{Y F} \\
\overrightarrow{\mathbf{e}}_{Z F}
\end{array}\right\} \\
\mathbf{G}_{E C E F 2 G C} & =\left[\begin{array}{ccc}
-\sin \phi & 0 & \cos \phi \\
0 & 1 & 0 \\
-\cos \phi & 0 & -\sin \phi
\end{array}\right] \cdot\left[\begin{array}{ccc}
\cos \theta & \sin \theta & 0 \\
-\sin \theta & \cos \theta & 0 \\
0 & 0 & 1
\end{array}\right]
\end{aligned}
$$

where $\theta$ is the longitude of the vehicle and $\phi$ is its declination. The geocentric frame is sometimes called the topocentric or local-vertical frame. The ECI, ECEF, and $G C$ frames are shown in Fig. 4.

A frame similar to the $G C$ frame is the geodetic $(G D)$ frame, sometimes known as the topodetic, localhorizontal, or North-Earth-Down $(N E D)$ frame. This frame is one in which the origin is located at the center of mass of the vehicle, and the $x$-axis, $\overrightarrow{\mathbf{e}}_{X D}$, points in the direction tangent to the surface of the Earth, in the direction of North. The $z$-axis, $\overrightarrow{\mathbf{e}}_{Z D}$, is aligned perpendicular to the $x$-axis and oriented downward, while the $y$-axis, $\overrightarrow{\mathbf{e}}_{Y D}$ is shared with the $G C$ frame. The $G D$ frame may be related to the $G C$ frame by the 


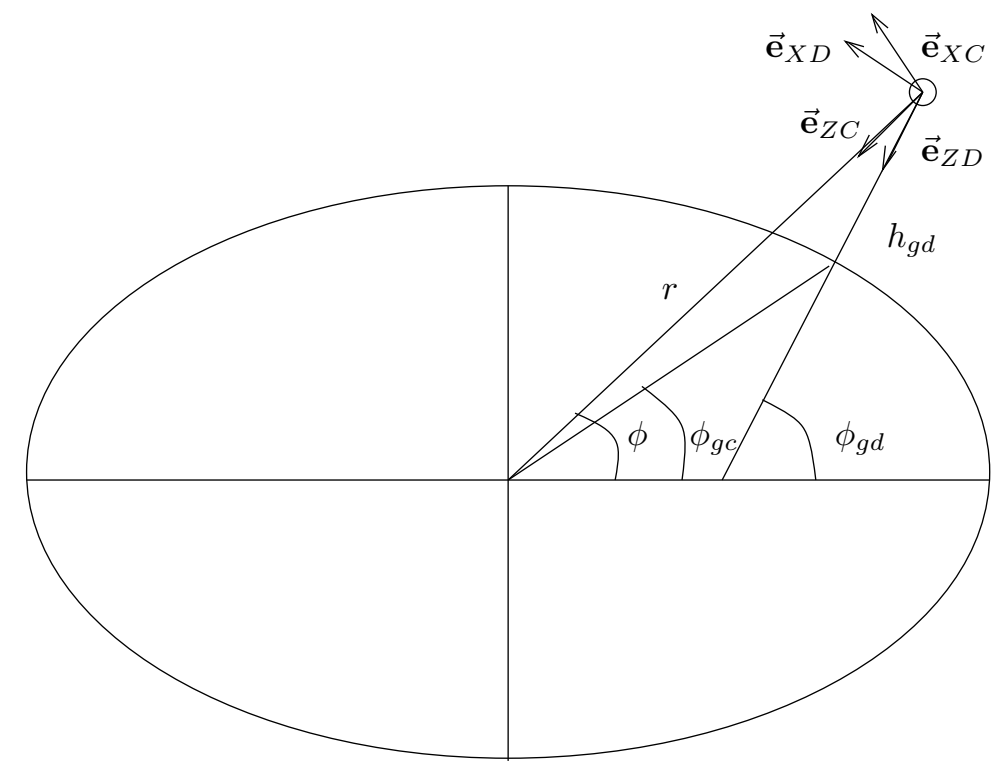

Figure 5. Declination, Geocentric Latitude, Geodetic Latitude, Geodetic Frame, and Geocentric Frame

transformation

$$
\begin{aligned}
\left\{\begin{array}{c}
\overrightarrow{\mathbf{e}}_{X D} \\
\overrightarrow{\mathbf{e}}_{Y D} \\
\overrightarrow{\mathbf{e}}_{Z D}
\end{array}\right\} & =\mathbf{G}_{G C 2 G D}\left\{\begin{array}{l}
\overrightarrow{\mathbf{e}}_{X C} \\
\overrightarrow{\mathbf{e}}_{Y C} \\
\overrightarrow{\mathbf{e}}_{Z C}
\end{array}\right\} \\
\mathbf{G}_{G C 2 G D} & =\left[\begin{array}{ccc}
\cos \epsilon & 0 & \sin \epsilon \\
0 & 1 & 0 \\
-\sin \epsilon & 0 & \cos \epsilon
\end{array}\right]
\end{aligned}
$$

where $\epsilon=\phi_{g d}-\phi$, with $\phi_{g d}$ being the geodetic latitude of the vehicle's sub-latitude point, which is the projection of the vehicle position to the surface of the Earth along the $\overrightarrow{\mathbf{e}}_{Z D}$ axis. The geodetic latitude is then the angle between the $\overrightarrow{\mathbf{e}}_{Z D}$ axis and the equatorial plane of the Earth. The $G C$ and $G D$ frames are shown in Fig. 5.

An alternate measure of the location of the sub-latitude point is the geocentric latitude, $\phi_{g c}$, which is the angle from the equatorial plane of the Earth to the line connecting the sub-latitude point and the center of the Earth. Fig. 5 shows the differences between the declination, geocentric latitude, and geodetic latitude for a planet with exaggerated eccentricity. Note that the three angles are equal for a spherical planet. Fig. 5 also shows the physical meaning of the geodetic altitude, $h_{g d}$, and the magnitude of the radius vector, $r-$ two variables that will be used presently.

Table 1. Summary of WGS-84 Earth Model Constants

\begin{tabular}{lc}
\hline \hline Constant & Value \\
\hline$R_{\oplus}$ & $2.092565 \cdot 10^{7} \mathrm{ft}$ \\
$e_{\oplus}$ & $8.1819191 \cdot 10^{-2}$ \\
$\mu$ & $1.4076444 \cdot 10^{16} \mathrm{ft}^{3} / \mathrm{s}^{2}$ \\
$J_{2}$ & $1.0826230 \cdot 10^{-3}$ \\
$\Omega$ & $4.1780741 \cdot 10^{-3} \circ / \mathrm{s}$ \\
\hline \hline
\end{tabular}

The relationship between the geocentric and geodetic latitude is quite simple, and takes the form

$$
\tan \phi_{g c}=\left(1-e_{\oplus}^{2}\right) \tan \phi_{g d}
$$


where $e_{\oplus}$ is the Earth's eccentricity. The transformation between declination and geodetic latitude, however, is more complicated. Vallado ${ }^{35}$ gives the formulas

$$
\begin{aligned}
\tan \phi_{g d} & =\tan \phi+\frac{R_{\oplus} e_{\oplus}^{2} \sin \phi_{g d}}{r \cos \phi \sqrt{1-e_{\oplus}^{2} \sin ^{2} \phi_{g d}}} \\
h_{g d} & =\frac{r \cos \phi}{\cos \phi_{g d}}-\frac{R_{\oplus}}{\sqrt{1-e_{\oplus}^{2} \sin ^{2} \phi_{g d}}}
\end{aligned}
$$

where $R_{\oplus}$ is the mean equatorial radius of the planet. Sofair ${ }^{36,37}$ determined an exact solution of Eqs. (21$22), \phi_{g d}(r, \phi)$ and $h_{g d}(r, \phi)$. The solution is explicit, and is free of singularities at the poles and the equator, although the solution space is confined to a region outside a spheroid fixed at the center of the Earth with semimajor axis of approximately $1.42 \times 10^{5} \mathrm{ft}$. This restriction is of no concern for the present problem as the region is well within the surface of the Earth.

The transformation to geocentric coordinates is much less complicated, and may be determined directly from Eqs. (21) and (22). Note that there are other definitions of the geocentric coordinates available in the literature. For an example, see Ref. 38.

The values of $R_{\oplus}, e_{\oplus}$, and other constants that describe the shape, mass, and rotation of the Earth are summarized in Table 1. These values are the World Geodetic System of 1984 (WGS-84). ${ }^{39}$

Lastly, the body $(B)$ frame is fixed to the center of mass of the vehicle with the $\overrightarrow{\mathbf{e}}_{X B}$ axis directed through the nose of the vehicle, the $\overrightarrow{\mathbf{e}}_{Z B}$ axis oriented perpendicular to the $\overrightarrow{\mathbf{e}}_{X B}$ axis, in the plane of symmetry, with the positive direction taken to be toward the bottom of the vehicle, and the $\overrightarrow{\mathbf{e}}_{Y B}$ axis completing the righthanded system so that it points out the right side of the vehicle. The $B$ frame is shown in Fig. 3 . The orientation of the $B$ frame is typically expressed by a 3-2-1 Euler angle sequence relating the $G D$ frame to the $B$ frame. The transformation is given by

$$
\begin{aligned}
\left\{\begin{array}{l}
\overrightarrow{\mathbf{e}}_{X B} \\
\overrightarrow{\mathbf{e}}_{Y B} \\
\overrightarrow{\mathbf{e}}_{Z B}
\end{array}\right\} & =\mathbf{G}_{G D 2 B}\left\{\begin{array}{l}
\overrightarrow{\mathbf{e}}_{X D} \\
\overrightarrow{\mathbf{e}}_{Y D} \\
\overrightarrow{\mathbf{e}}_{Z D}
\end{array}\right\} \\
\mathbf{G}_{G D 2 B} & =\left[\begin{array}{ccc}
1 & 0 & 0 \\
0 & \cos \Phi & \sin \Phi \\
0 & -\sin \Phi & \cos \Phi
\end{array}\right] \cdot\left[\begin{array}{ccc}
\cos \Theta & 0 & -\sin \Theta \\
0 & 1 & 0 \\
\sin \Theta & 0 & \cos \Theta
\end{array}\right] \cdot\left[\begin{array}{ccc}
\cos \Psi & \sin \Psi & 0 \\
-\sin \Psi & \cos \Psi & 0 \\
0 & 0 & 1
\end{array}\right]
\end{aligned}
$$

where $\Psi, \Theta$, and $\Phi$ are the azimuth, elevation, and bank angles, respectively, also commonly referred to as the yaw, pitch, and roll angles.

Although the Euler angle sequence offers an ease of visualization, other parameterizations of the vehicle orientation are preferable for implementation in the equations of motion and filtering algorithms. ${ }^{40}$ The most advantageous of these parameterizations are the Euler parameters, the use of which leads to a representation of the attitude transformation matrix of the form

$$
\mathbf{G}_{G D 2 B}=\left[\begin{array}{ccc}
e_{0}^{2}+e_{1}^{2}-e_{2}^{2}-e_{3}^{2} & 2\left(e_{1} e_{2}+e_{0} e_{3}\right) & 2\left(e_{1} e_{3}-e_{0} e_{2}\right) \\
2\left(e_{1} e_{2}-e_{0} e_{3}\right) & e_{0}^{2}-e_{1}^{2}+e_{2}^{2}-e_{3}^{2} & 2\left(e_{0} e_{1}+e_{2} e_{3}\right) \\
2\left(e_{1} e_{3}+e_{0} e_{2}\right) & 2\left(e_{2} e_{3}-e_{0} e_{1}\right) & e_{0}^{2}-e_{1}^{2}-e_{2}^{2}+e_{3}^{2}
\end{array}\right]
$$

The Euler parameters constitute a four parameter set that describe the orientation of the $B$ frame with respect to the $G D$ frame. Due to the fact that four parameters are used to describe a rotation with three degrees of freedom, the constraint equation $e_{0}^{2}+e_{1}^{2}+e_{2}^{2}+e_{3}^{2}=1$ must also be satisfied.

\section{B. Equations of Motion}

Newton's laws of motion, expressed for a body moving relative to a planet-fixed reference frame, lead to the translational equations of motion of the form ${ }^{24}$

$$
\begin{aligned}
\dot{r} & =-w \\
\dot{\phi} & =\frac{u}{r} \\
\dot{\theta} & =\frac{v}{r \cos \phi}-\Omega
\end{aligned}
$$




$$
\begin{aligned}
\dot{u} & =a_{X C}+\frac{1}{r}\left(u w-v^{2} \tan \phi\right)-\frac{3 \mu J_{2}}{2 r^{4}} \sin (2 \phi) \\
\dot{v} & =a_{Y C}+\frac{1}{r}(u v \tan \phi+v w) \\
\dot{w} & =a_{Z C}-\frac{1}{r}\left(u^{2}+v^{2}\right)+\frac{\mu}{r^{2}}-\frac{3 \mu J_{2}}{2 r^{4}}\left(2-3 \cos ^{2} \phi\right)
\end{aligned}
$$

where $u, v$, and $w$ are the $x, y$, and $z$-axis components of the velocity in the $G C$ frame, respectively, and $a_{X C}, a_{Y C}, a_{Z C}$ are the geocentric representation of the accelerations acting at the body center of mass, not including the gravitational terms.

These accelerations may be given in terms of the body-axis accelerations at the IMU location by the transformations

$$
\left\{\begin{array}{c}
a_{X B} \\
a_{Y B} \\
a_{Z B}
\end{array}\right\}=\left\{\begin{array}{c}
a_{X T} \\
a_{Y T} \\
a_{Z T}
\end{array}\right\}-\left(\boldsymbol{\Gamma}^{2}+\dot{\boldsymbol{\Gamma}}\right)\left\{\begin{array}{c}
x_{M} \\
y_{M} \\
z_{M}
\end{array}\right\}-2 \boldsymbol{\Gamma}\left\{\begin{array}{c}
\dot{x}_{M} \\
\dot{y}_{M} \\
\dot{z}_{M}
\end{array}\right\}-\left\{\begin{array}{c}
\ddot{x}_{M} \\
\ddot{y}_{M} \\
\ddot{z}_{M}
\end{array}\right\}
$$

where $a_{X B}, a_{Y B}$, and $a_{Z B}$ are the accelerations acting at the vehicle center of mass, represented in the $B$ frame, $a_{X T}, a_{Y T}$, and $a_{Z T}$ are the accelerations acting at the IMU location, expressed in a frame parallel to the $B$ frame, $x_{M}, y_{M}$, and $z_{M}$ are the components of the position of the IMU measured from the vehicle center of mass, expressed in the $B$ frame, and

$$
\boldsymbol{\Gamma}=\left[\begin{array}{ccc}
0 & -R & Q \\
R & 0 & -P \\
-Q & P & 0
\end{array}\right]
$$

where $P, Q$, and $R$ and the components of the angular velocity of the $B$ frame with respect to the $E C I$ frame, expressed in the $B$ frame.

The accelerations may then be transformed into the $G C$ frame to be consistent with the equations of motion by means of

$$
\left\{\begin{array}{l}
a_{X C} \\
a_{Y C} \\
a_{Z C}
\end{array}\right\}=\mathbf{G}_{B 2 G C}\left\{\begin{array}{l}
a_{X B} \\
a_{Y B} \\
a_{Z B}
\end{array}\right\}
$$

where $\mathbf{G}_{B 2 G C}=\left(\mathbf{G}_{G D 2 B} \mathbf{G}_{G C 2 G D}\right)^{T}$.

The rotational kinematics of a vehicle relative to the $G D$ frame may be written as

$$
\left\{\begin{array}{c}
\dot{e}_{0} \\
\dot{e}_{1} \\
\dot{e}_{2} \\
\dot{e}_{3}
\end{array}\right\}=\frac{1}{2}\left[\begin{array}{ccc}
-e_{1} & -e_{2} & -e_{3} \\
e_{0} & -e_{3} & e_{2} \\
e_{3} & e_{0} & -e_{1} \\
-e_{2} & e_{1} & e_{0}
\end{array}\right]\left(\left\{\begin{array}{c}
P \\
Q \\
R
\end{array}\right\}-\frac{1}{r} \mathbf{G}_{G C 2 B}\left\{\begin{array}{c}
v \\
-u \\
-v \tan \phi
\end{array}\right\}\right)
$$

In summary, the equations of motion for a vehicle relative to a rotating central body are given by the combination of Eqs. (26-31) and (35), together with the transformation given in Eq. (32). The states may be written as $\mathbf{x}=\left\{\begin{array}{llllllllll}r & \phi & \theta & u & v & w & e_{0} & e_{1} & e_{2} & e_{3}\end{array}\right\}^{T}$. The strapdown formulation of the inertial measurements in the equations of motion may be modified without difficulty to a platform implementation as in Ref. 41 for applications that make use of that particular type of inertial measurement source.

The dynamic model discussed in this section is similar to that implemented in the original STEP code with several subtle differences. The first and most obvious difference is that the current implementation uses the radius of the vehicle position relative to the planet center of mass whereas the STEP equations of motion used an altitude variable. The current formulation is superior in the sense that additional transformations between altitude and radius as required to compute the gravitational acceleration is not required. Another difference between the current dynamic model and the STEP dynamic model is that the latter did not distinguish between the $G D$ frame and the $G C$ frame as does the former. Although the difference between these two frames is a small, it is not negligible, and may lead to large errors in the north and down velocity components, as was demonstrated in Ref. 42.

It should also be noted that the equations of motion developed in this section make use the Euler parameters to describe the orientation of the $B$-frame relative to the $G D$-frame, as was used in STEP as 
well. Recent research has shown that some problems exist when using this particular parameterization within estimation algorithms, which lead to the possibility of ill-conditioned covariance matrices due to the constraint on the norm of the Euler parameters. ${ }^{43-45}$ Pittelkau, ${ }^{46}$ however, showed that filters with properly tuned process noise models do not suffer from these problems, and that enforcing the Euler parameter constraint within the filter update equation is not an ad-hoc modification of the filter, but in fact equivalent to a measurement update equation. Therefore, the use of the Euler parameter set within the estimation procedure remains justified.

The next section discusses the development of the equations relating the states to the measurement data for use in the filtering process.

\section{Measurement Equations}

This section discusses the formulation of the measurement equations used within the trajectory reconstruction tool, including the modelling of various error sources. The first such measurement equation is that of the GPS, or some other source such as a radar altimeter, that provides redundant position information that may be incorporated into the trajectory estimate during the filtering process. Secondly, a GPS unit or other sources may provide a velocity measurement, which may also be incorporated into the trajectory estimation procedure. Another source of interest is the IMU data, which records the acceleration and angular rates of the vehicle in question. This data source in not used to provide measurement updates via a filtering algorithm, but are inputs into the equations of motion that are integrated to provide the state predictions at the time of the measurement update. However, a vehicle that contains two separate IMUs could use one to process the equations of motion, and the other to provide redundant data for use in filtering. Also included in this section are equations for the measurement of the vehicle orientation, and for ground-based radar tracking stations.

\section{A. Position Measurement Equations}

The filtering algorithms require observations of the vehicle state in order to generate a minimum variance trajectory estimate. One such source of the observation data is a satellite navigation Global Positioning System (GPS) receiver carried within the vehicle. The GPS-based observations provide position estimates of the receiver location.

The position observation data, provided in the geodetic coordinates $h_{g d_{M}}, \phi_{g d_{M}}$, and $\theta_{g d_{M}}$, can be written as a function of the vehicle state by the transformations

$$
\begin{aligned}
\left\{\begin{array}{c}
x^{\star} \\
y^{\star} \\
z^{\star}
\end{array}\right\} & =\mathbf{G}_{B 2 E C E F}\left\{\begin{array}{l}
x_{G}+\delta_{X G} \\
y_{G}+\delta_{Y G} \\
z_{G}+\delta_{Z G}
\end{array}\right\} \\
x_{F_{G}} & =r \cos \phi \cos \theta+x^{\star} \\
y_{F_{G}} & =r \cos \phi \sin \theta+y^{\star} \\
z_{F_{G}} & =r \sin \phi+z^{\star} \\
r_{G} & =\sqrt{x_{F_{G}}^{2}+y_{F_{G}}^{2}+z_{F_{G}}^{2}} \\
\sin \phi_{G} & =\left(\frac{z_{F_{G}}}{r_{G}}\right) \\
\cos \theta_{G} & =\frac{x_{F_{G}}}{r_{G} \cos \phi_{G}} \\
\sin \theta_{G} & =\frac{y_{F_{G}}}{r_{G} \cos \phi_{G}} \\
h_{g d_{M}} & =h_{g d}\left(r_{G}, \phi_{G}\right) \\
\phi_{g d_{M}} & =\phi_{g d}\left(r_{G}, \phi_{G}\right)
\end{aligned}
$$

where $x_{G}, y_{G}$, and $z_{G}$ are the components of the position of the GPS unit relative to the vehicle center of mass, expressed in the B frame (with bias error sources $\delta_{X G}, \delta_{Y G}$, and $\delta_{Z G}$ ), while $x^{\star}, y^{\star}$, and $z^{\star}$ are the same position expressed in the ECEF frame. The variables $x_{F_{G}}, y_{F_{G}}$, and $z_{F_{G}}$ represent the ECEF coordinates of the GPS receiver, $r_{G}, \phi_{G}$, and $\theta_{G}$ are the geocentric coordinates of the GPS receiver, and $h_{g d_{M}}$ and $\phi_{g d_{M}}$ are the position measurements, calculated using Sofair's method. 


\section{B. Velocity Measurement Equations}

Similarly, the velocity of the GPS unit or other observation is provided in the planet-relative, geodetic velocities $u_{g d_{M}}, v_{g d_{M}}$, and $w_{g d_{M}}$, and may be found as a function of the inertial velocity of the vehicle center of mass, in the GC frame, by first transforming the inertial velocity from the GC frame to the GD frame, and then subtracting out the velocity due to the planetary rotation in order to create planet-relative velocities. Lastly, the velocity due to the rotation of the body and the offset of the GPS unit from the vehicle center of mass must also be accounted for. These transformations take the form

$$
\left\{\begin{array}{c}
u_{g d_{M}} \\
v_{g d_{M}} \\
w_{g d_{M}}
\end{array}\right\}=\mathbf{G}_{G C 2 G D}\left\{\begin{array}{c}
u \\
v-r \Omega \cos \phi \\
w
\end{array}\right\}+\mathbf{G}_{B 2 G D} \boldsymbol{\Gamma}\left\{\begin{array}{c}
x_{G}+\delta_{X G} \\
y_{G}+\delta_{Y G} \\
z_{G}+\delta_{Z G}
\end{array}\right\}
$$

\section{Acceleration and Angular Rate Measurement Equations}

The equations of motion for a vehicle, developed in Sec. III B, assume that the acceleration and angular rates of the vehicle center of mass are known functions of time. The inertial flight data contains this information, however, several transformations are required in order to bring this data into the proper form, and furthermore, the data recorded during the flight is subject to systematic errors and measurement noise. Systematic error sources may be modelled, and if values are known, their effects may be compensated for in the trajectory reconstruction procedure. Otherwise, the values of the systematic errors may be estimated using the filtering techniques, with an augmented state vector consisting of the usual position, velocity, and attitude states, along with the systematic error terms that are assumed to be constants over the time interval in question.

Assuming small systematic error sources allows for a linear representation of the IMU measurement equations of the form ${ }^{16,47,48}$

$$
\left\{\begin{array}{c}
a_{X M} \\
a_{Y M} \\
a_{Z M}
\end{array}\right\}=\left[\begin{array}{ccc}
1+\xi_{X} & \mu_{X Y} & \mu_{X Z} \\
\mu_{Y X} & 1+\xi_{Y} & \mu_{Y Z} \\
\mu_{Z X} & \mu_{Z Y} & 1+\xi_{Z}
\end{array}\right]\left\{\begin{array}{c}
a_{X T} \\
a_{Y T} \\
a_{Z T}
\end{array}\right\}+\left\{\begin{array}{c}
\eta_{X} \\
\eta_{Y} \\
\eta_{Z}
\end{array}\right\}
$$

where $a_{X T}, a_{Y T}$, and $a_{Z T}$ are the true accelerations at the IMU location, expressed in the B frame, and $a_{X M}, a_{Y M}$, and $a_{Z M}$ are the measured accelerations at the IMU location, expressed in a frame parallel to the B frame. The parameters $\xi_{X}, \xi_{Y}$, and $\xi_{Z}$ account for the scale factor error in the accelerometer data, $\mu_{X Y}, \mu_{X Z}, \mu_{Y X}, \mu_{Y Z}, \mu_{Z X}$, and $\mu_{Z Y}$ account for misalignment errors. The parameters $\eta_{X}, \eta_{Y}$, and $\eta_{Z}$ account for bias. The components of the position of the IMU with respect to the vehicle center of mass $\left(x_{M}\right.$, $y_{M}$, and $z_{M}$ ) are also assumed to be perturbed by the biases $\delta_{X M}, \delta_{Y M}$, and $\delta_{Z M}$, respectively.

Similarly, the inertial angular velocity components are subject to systematic errors of the form ${ }^{16,47,48}$

$$
\left\{\begin{array}{c}
P_{M} \\
Q_{M} \\
R_{M}
\end{array}\right\}=\left[\begin{array}{ccc}
1+\xi_{P} & \mu_{P Q} & \mu_{P R} \\
\mu_{Q P} & 1+\xi_{Q} & \mu_{Q R} \\
\mu_{R P} & \mu_{R Q} & 1+\xi_{R}
\end{array}\right]\left\{\begin{array}{c}
P \\
Q \\
R
\end{array}\right\}+\left\{\begin{array}{c}
\eta_{P} \\
\eta_{Q} \\
\eta_{R}
\end{array}\right\}
$$

where $P_{M}, Q_{M}$, and $R_{M}$ are the measured inertial angular velocity components, expressed in the $\mathrm{B}$ frame, and $P, Q$, and $R$ are the true inertial angular velocity components of the vehicle, expressed in the $\mathrm{B}$ frame. $\xi_{P}, \xi_{Q}$, and $\xi_{R}$ are the scale factor errors, $\mu_{P Q}, \mu_{P R}, \mu_{Q P}, \mu_{Q R}, \mu_{R P}, \mu_{R Q}$ are the misalignment errors, and $\eta_{P}, \eta_{Q}$, and $\eta_{R}$ are the bias terms. Note that the inertial angular velocity is a property of the body and not a point, and so these terms do not require a moment-arm transformation as do the accelerations.

Note that the preceding transformations give the measured acceleration and angular rate data as a function of the true acceleration and angular rates, thus, in order to provide the proper inputs into the equations of motion, the IMU measurement equations must be inverted to yield the true acceleration as a function of the measured acceleration and error sources, either known or estimated.

The acceleration measurement error equations developed in this section are used principally to correct the forcing function input to the equations of motion. However, a vehicle with two or more IMUs could potentially use the additional data as a source of redundancy, which may be incorporated into the state estimation in a manner similar to the position and velocity measurements. 


\section{Radar Measurement Equations}

The last measurement source of interest to the current document is that associated with ground-based radar tracking stations that measure the range, azimuth, and elevation of the vehicle with respect to a $G D$-frame local to the tracking station. Given the site coordinates $h_{g d_{S}}, \phi_{g d_{S}}, \theta_{S}$, or equivalently, $r_{S}, \phi_{S}$ and $\theta_{S}$, the relative position between the vehicle and the tracking station, expressed in a $G D$-frame local to the station, may be expressed as

$$
\left\{\begin{array}{c}
x_{r e l} \\
y_{r e l} \\
z_{r e l}
\end{array}\right\}=\mathbf{G}_{S}\left\{\begin{array}{c}
r \cos \phi \cos \theta-r_{S} \cos \phi_{S} \cos \theta_{S} \\
r \cos \phi \sin \theta-r_{S} \cos \phi_{S} \sin \theta_{S} \\
r \sin \phi-r_{S} \sin \phi_{S}
\end{array}\right\}
$$

where

$$
\mathbf{G}_{S}=\left[\begin{array}{ccc}
-\sin \phi_{g d_{S}} & 0 & \cos \phi_{g d_{S}} \\
0 & 1 & 0 \\
-\cos \phi_{g d_{S}} & 0 & -\sin \phi_{g d_{S}}
\end{array}\right] \cdot\left[\begin{array}{ccc}
\cos \theta_{S} & \sin \theta_{S} & 0 \\
-\sin \theta_{S} & \cos \theta_{S} & 0 \\
0 & 0 & 1
\end{array}\right]
$$

The range, azimuth, and elevation angles may then be written as

$$
\begin{aligned}
\nu & =\sqrt{x_{r e l}^{2}+y_{r e l}^{2}+z_{r e l}^{2}} \\
\sin \chi & =-\frac{z_{r e l}}{\nu} \\
\sin \kappa & =\frac{y_{r e l}}{\sqrt{x_{r e l}^{2}+y_{r e l}^{2}}} \\
\cos \kappa & =\frac{x_{r e l}}{\sqrt{x_{r e l}^{2}+y_{r e l}^{2}}}
\end{aligned}
$$

where $\nu$ is the range measurement, $\chi$ is the elevation angle, and $\kappa$ is the azimuth angle, respectively.

\section{Application to the Analysis of Flight Data}

This section summarizes the results of applying the methods developed in the previous sections to the problem of estimating the trajectory of the Hyper-X flight test vehicle given the IMU data and several redundant data sources, such as GPS and radar measurements. The data preprocessing methods are discussed first.

\section{A. Flight Data Preprocessing}

This section summarizes the data preprocessing that were required in order to provide useful measurements as needed for the trajectory reconstruction process. The raw flight data were passed through a preprocessing tool, known as PREPR, ${ }^{49}$ which consists of four main utilities. The first utility removes duplicate points in the data series which result from differences between the sensor data refresh rate and the telemetry sampling rate. The second utility allows users to interactively remove rogue points from the data series. Users select individual or groups of points to remove from a graph of the data series. The third utility samples the data series at a uniform time step, using a user-specified option of linear, cubic spline, or Hermite spline interpolation between available data points. The fourth utility gives the user the option to filter the data series using Fourier series and optimal Weiner filtering method ${ }^{50}$ available within the Systems Identification Program for Aircraft (SIDPAC). ${ }^{51}$ A separate fifth utility is included which can be used to differentiate a data series once it has been passed through the previous four routines. The user is given the option to use backward difference or center divided difference methods. Alternatively, users can select to curve fit the data using either cubic or Hermite spline polynomials, and to individually differentiate each piecewise segment.

Data recorded by the Hyper-X control room were used for the drop phase up to the end of the engine test experiment. Some communications issues arose during the descent portion of the flight in which data were not transmitted to the control room, but were recorded by a P3 chase plane. The data from the control room and from the P3 chase plane were spliced together to give a continuous data stream for post-flight analysis. This spliced data set was used for the portion of the flight beginning just after the engine test to the end of the flight. 

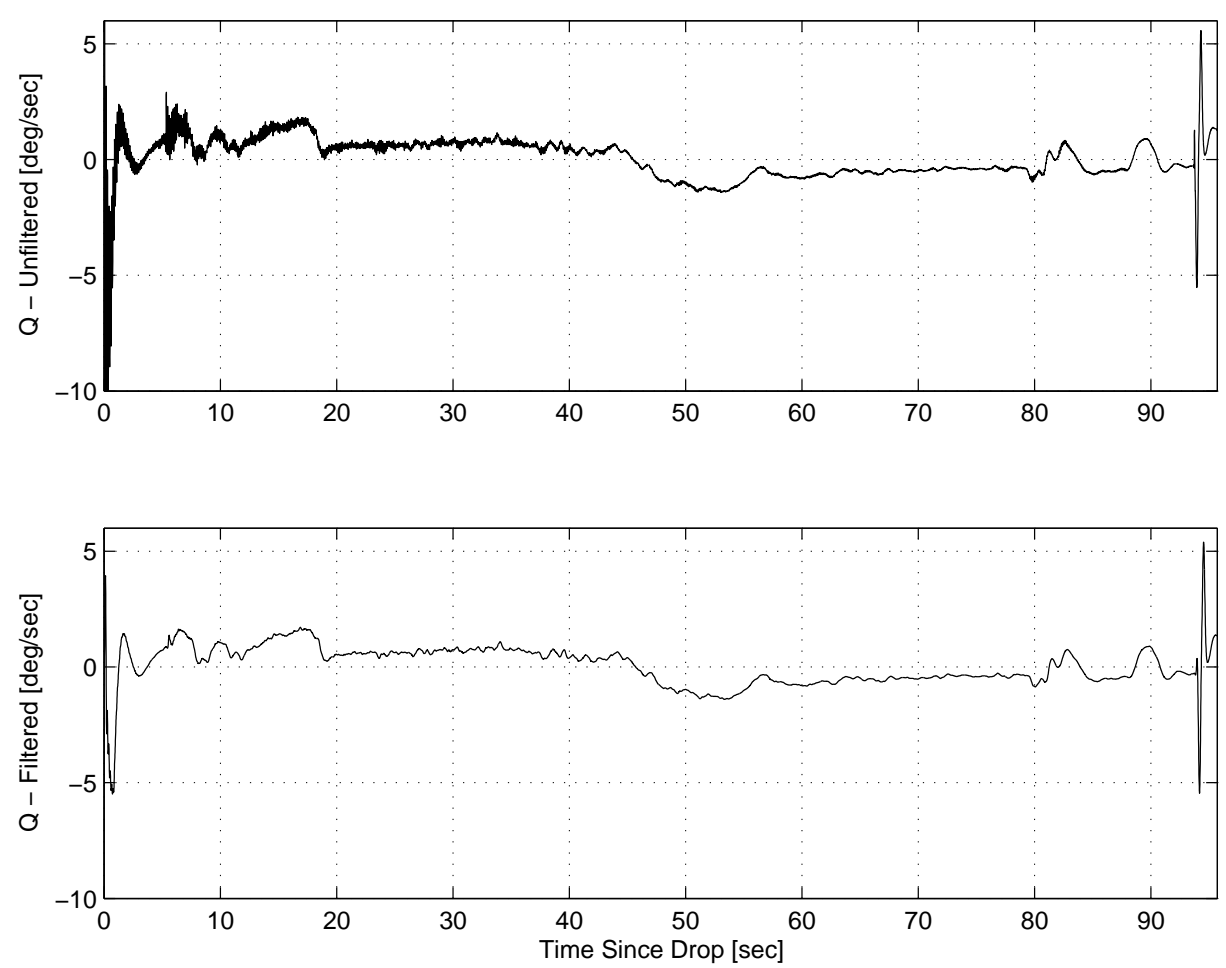

Figure 6. Body Y-Axis Inertial Angular Velocity

\section{Inertial Data}

Measurements of acceleration and angular rates were provided by QA2000 accelerometers and GG1320 Digital Laser Gyroscopes (DLG) contained within the Honeywell H-764G system, ${ }^{52}$ a unit with a background of numerous civilian and military applications. These measurement data were obtained from the on-board telemetry stream at a rate of $100 \mathrm{~Hz}$ and were first preprocessed by removing points which contained obviously bad or rogue data. Next, the data were edited to remove points which could be considered as repeated, stale, or non-updated frames. Gaps in the data due to telemetry dropouts or due to edited data were filled in by a piecewise cubic Hermite interpolating function, and were then filtered to remove the effects of high-frequency structural noise, measurement noise, and to smooth out the data from quantization effects.

Table 2. Summary of Cut-Off Frequencies for IMU Data Preprocessing

\begin{tabular}{lcc}
\hline \hline IMU Data & HXLV Stack Cut-Off & HXRV Cut-Off \\
\hline$a_{X M}$ & $10 \mathrm{~Hz}$ & $10 \mathrm{~Hz}$ \\
$a_{Y M}$ & $8 \mathrm{~Hz}$ & $10 \mathrm{~Hz}$ \\
$a_{Z M}$ & $8 \mathrm{~Hz}$ & $10 \mathrm{~Hz}$ \\
$P_{M}$ & $8 \mathrm{~Hz}$ & $10 \mathrm{~Hz}$ \\
$Q_{M}$ & $5 \mathrm{~Hz}$ & $10 \mathrm{~Hz}$ \\
$R_{M}$ & $8 \mathrm{~Hz}$ & $10 \mathrm{~Hz}$ \\
\hline \hline
\end{tabular}

Table 2 summarizes the cut-off frequencies used to filter the IMU data for the periods when the HXLV stack is complete and for the periods after the HXRV has separated from the HXLV. The body Y-Axis inertial angular velocity is shown in Fig. 6 as an example of the data before and after the filtering process, during the boost phase. In this example, evidence of a known $8 \mathrm{~Hz}$ structural mode in the HXLV configuration can clearly be seen, which influenced the decision to use a lower cut-off frequency for this channel. After the separation of the HXRV from the HXLV, the IMU data had almost no apparent structural mode content, 
and therefore a higher cut-off frequency was used for preprocessing.

Numerical derivatives of the inertial angular rates, as required for evaluation of Eq. (32), were calculated using a second-order centered divided difference scheme, except for the beginning and end points, which used first-order forward and backward divided differences, respectively.

Preliminary values of the process noise standard deviations used in connection with the inertial measurement data in the EKF formulation were taken from Refs. 53 and 54, which were tuned somewhat to suit the problem at hand.

\section{GPS Data}

GPS measurements were obtained as outputs from the H-764G GPS Embedded Module (GEM), which is a 5-channel receiver, operating in Precision Positioning Service (PPS) mode at a rate of $1 \mathrm{~Hz}$. The GPS data required little preprocessing, aside from extracting the data from the telemetry stream. There were several issues with the data itself, however. First, the east velocity channel saturated at approximately 28 sec. after the HXLV was dropped from the B-52 carrier vehicle, due to inadequate telemetry system bit assignments. The east velocity data unsaturated during the vehicle descent, at a time of approximately 448 sec. after drop. Secondly, the GPS antenna carried on the HXRV itself was activated at the instant of the drop (previous GPS points from the HXRV telemetry stream were provided by the vehicle flight management computer, but were derived from a GPS antenna carried on the B-52), and as a result, suspected blockage or multi path effects seem to have corrupted the first several data points recorded after the drop. Therefore, the first several points were deleted from the data set and were not considered in the reconstruction process. Also, there were several brief dropouts of all GPS data streams along the trajectory, as well as several stale frames and repeated time points during the descent, all of which were deleted from the data set.

The GPS measurement noise characteristics were taken from Ref. 54 and were scaled linearly by the Figure of Merit (FOM), which is a telemetry parameter that takes on a value depending on the estimated errors in the GPS-based navigation solution. The FOM depends on many factors including the number of satellites used to compute the navigation solution and the dilution of precision in the event of an unfavorable geometry between the satellites and the receiver.

\section{Radar Data}

Many radar stations were tasked to provide measurements of the Hyper-X vehicle during its flight both for range safety and for trajectory reconstruction purposes. This tracking data is summarized in Ref. 55 along with details of the corrections made for atmospheric refraction. The tracking data must be further corrected for unknown bias and scale factor errors in order to be useful for trajectory reconstruction.

During the captive carry portion of the flight, highly accurate GPS-aided INS navigation solutions are available in terms of geodetic altitude, geodetic latitude, and longitude. The radar tracking data are also available during the same portion of the flight, providing position measurements of the target relative to the tracking station, in terms of range, azimuth, and elevation measurements. The GPS/INS data may be related to the radar tracking data by means of the transformations given in Sec. IV D. A simple systematic error model relating measured quantities $\left(\nu_{m}, \kappa_{m}\right.$, and $\left.\chi_{m}\right)$ to the true quantities $(\nu, \kappa$, and $\chi)$ for the tracking data is of the form

$$
\begin{aligned}
\nu_{m} & =\left(1+\xi_{\nu}\right) \nu+\eta_{\nu} \\
\kappa_{m} & =\left(1+\xi_{\kappa}\right) \kappa+\eta_{\kappa} \\
\chi_{m} & =\left(1+\xi_{\chi}\right) \chi+\eta_{\chi}
\end{aligned}
$$

where $\xi_{\nu}, \xi_{\kappa}$, and $\xi_{\chi}$ are the scale factor errors associated with the range, azimuth, and elevation data, and $\eta_{\nu}, \eta_{\kappa}$, and $\eta_{\chi}$ are bias errors. By defining the radar error parameter state space as

$$
\mathbf{x}_{r}=\left\{\begin{array}{llllll}
\xi_{\nu} & \xi_{\kappa} & \xi_{\chi} & \eta_{\nu} & \eta_{\kappa} & \eta_{\chi}
\end{array}\right\}^{T}
$$

the model for the measurement equation in terms of the navigation data may be written as

$$
\mathbf{g}_{r}\left(\mathbf{x}_{r}, \mathbf{w}_{r}, t\right)=\left[\begin{array}{cccccc}
\nu_{\text {nav }} & 0 & 0 & 1 & 0 & 0 \\
0 & \kappa_{\text {nav }} & 0 & 0 & 1 & 0 \\
0 & 0 & \chi_{\text {nav }} & 0 & 0 & 1
\end{array}\right] \mathbf{x}_{r}+\left[\begin{array}{cccccc}
1 & 0 & 0 & 1 & 0 & 0 \\
0 & 1 & 0 & 0 & 1 & 0 \\
0 & 0 & 1 & 0 & 0 & 1
\end{array}\right] \mathbf{w}_{r}+\left\{\begin{array}{c}
\nu_{\text {nav }} \\
\kappa_{\text {nav }} \\
\chi_{\text {nav }}
\end{array}\right\}
$$



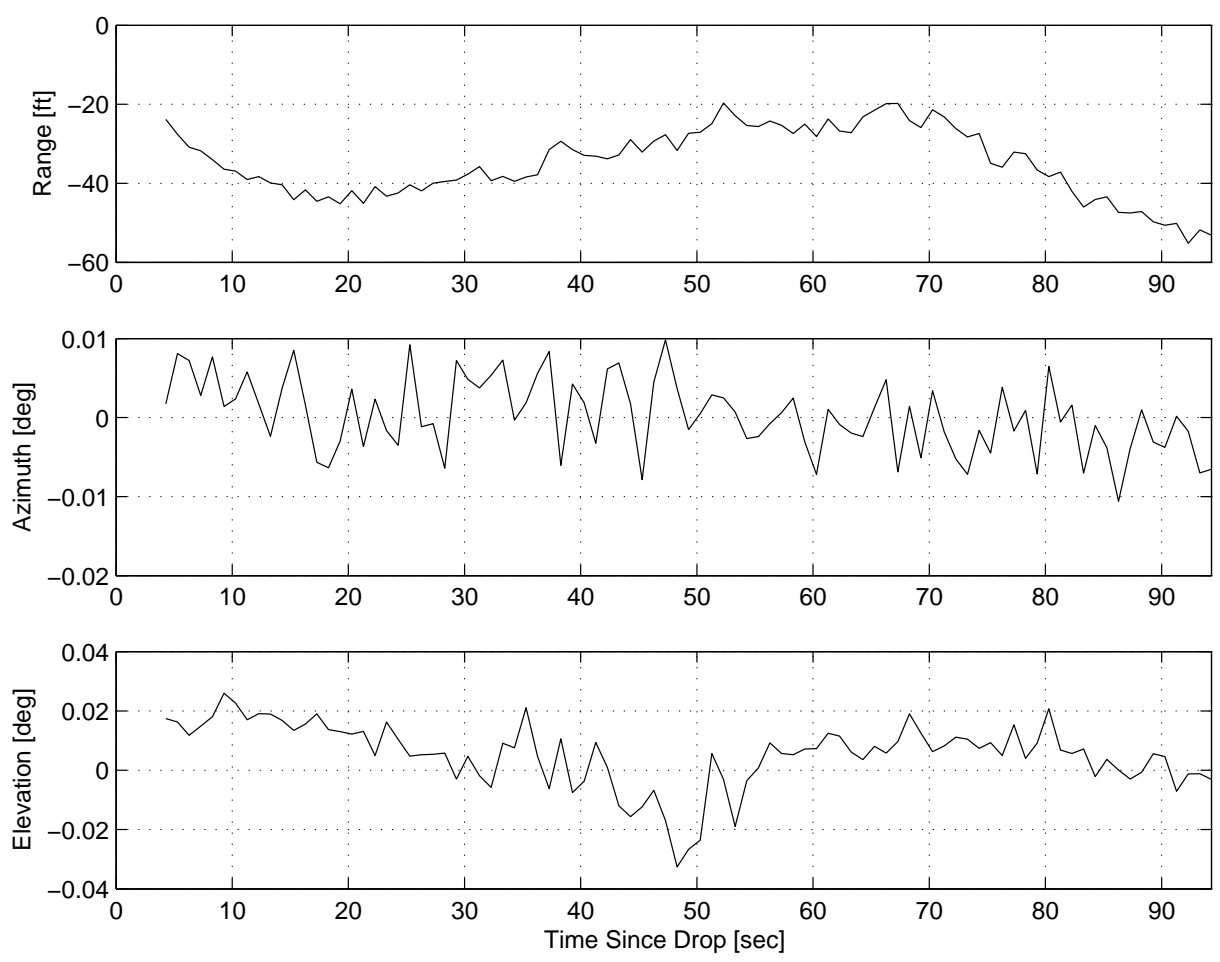

Figure 7. Radar and GPS Residuals as Seen by MOTR-16

where $\nu_{n a v}, \kappa_{n a v}$, and $\chi_{n a v}$ are the range, azimuth, and elevation as computed from the navigation solution, $\mathbf{w}_{r}=\left\{\begin{array}{llllll}w_{\nu_{\text {nav }}} & w_{\kappa_{\text {nav }}} & w_{\chi_{\text {nav }}} & w_{\nu} & w_{\kappa} & w_{\chi}\end{array}\right\}^{T}$ represents the zero-mean uncertainty associated with the range, azimuth, and elevation as computed from the INS data (essentially the navigation uncertainty transformed into range, azimuth, and elevation coordinates) and the measurement noise uncertainty for the radar measurements themselves. These uncertainties may be characterized in terms of their covariance matrix, $\mathbf{R}_{r}=E\left(\mathbf{w}_{r} \mathbf{w}_{r}^{T}\right)$.

The error parameters were estimated by using a Kalman filter implementation, as is described in Sec. II A. (A similar method for radar calibration using GPS measurements was used in Ref. 56 for air traffic control applications.) The resulting minimum-variance estimates of the error parameters were then applied to the rest of the radar tracking data in order to provide calibrated measurements of the vehicle trajectory during the flight experiment. Unfortunately, the tracking data provided were at low elevation angles and as a result, suffered from high noise content, atmospheric, and nonlinear effects that could not be accounted for in the calibration method. Therefore, the radar data was deemed to be unsuitable for direct use in the trajectory reconstruction. The data did prove useful, however, for providing reasonableness checks for the GPS data.

As an example of the reasonableness checks, consider the MOTR-16 tracking station, located at Vandenberg Air Force Base (VAFB). This station provided tracking measurements on a C-Band transponder located on the starboard side of the HXRV. The GPS measurements of altitude, latitude, and longitude may be transformed into range, azimuth, and elevation data, as would be seen from this tracking station. Fig. 7 shows the residuals between the transformed GPS data and the calibrated radar measurements. Although the range and elevation residuals seem to have some systematic errors (i.e., nonlinearity), and results of the comparison are noisy, the plots serve to show that the residuals between the GPS-derived quantities and the radar measurements do not seem to change in character between periods of relatively benign flight, such as that seen by the Hyper-X vehicle shortly after drop, and periods of high Mach number and high acceleration such as that encountered toward the end of the boost segment. Therefore, these results serve to give some credibility to the use of the GPS data for trajectory reconstruction purposes. 


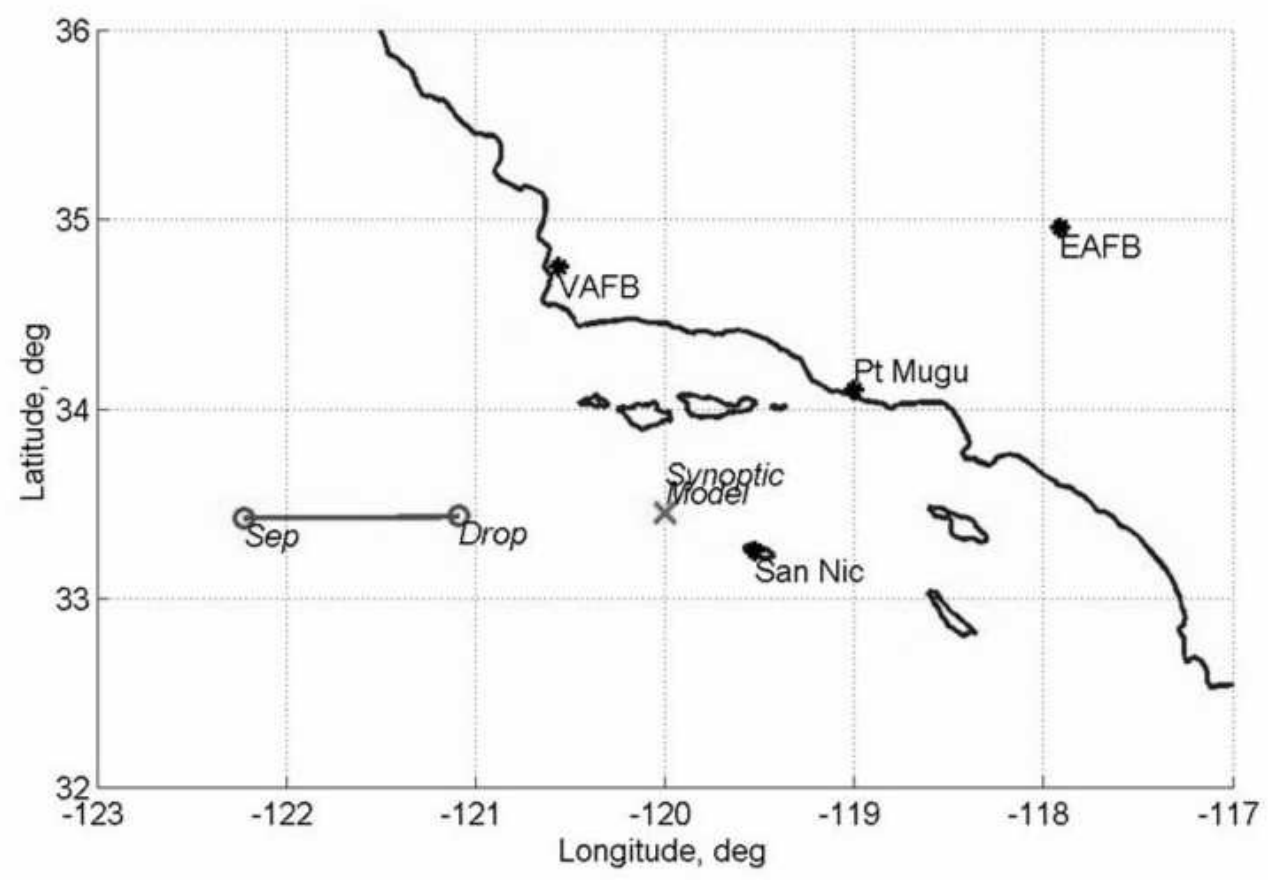

Figure 8. Synoptic Weather Model

\section{B. Atmosphere Reconstruction}

Unlike the planet-relative trajectory reconstruction, a typical atmosphere reconstruction relies solely upon measurements taken with large space and time separations between the measurement point and the vehicle during its flight. This fact is no different for the case of the Hyper-X flight. A total of 16 weather balloons were launched in order to provide the atmospheric data required to compute parameters of interest that are not estimated in the trajectory reconstruction (since the methods reconstruct a planet-relative trajectory), such as the dynamic pressure, Mach number, and so on. A total of 4 balloons were launched from each of Edwards Air Force Base (EAFB), VAFB, Point Magu Naval Air Weapons Center, and San Nicolas Island Naval Station. The map in Fig. 8 shows the separation of the balloon launch sites from the HXLV drop site. The balloon data was assembled into a synoptic model containing the ambient atmospheric properties as a function of altitude above the point $\phi_{g d_{r e f}}=33.45^{\circ}, \theta_{\text {ref }}=-120.0^{\circ}$, which is marked by the $X$ on the map in Fig. 8. The HXLV stack drop and HXLV/HXRV separation points are also shown for reference. Weather data at a point other than the location of the synoptic model reference point must be transformed by making use of pressure gradient information to map the pressure to such a location. The temperature table is assumed to be invariant with respect to latitude and longitude, and the density follows directly from the application of the equation of state.

\section{Results of the Trajectory Reconstruction}

The results of the application of the EKF method to the Hyper-X trajectory reconstruction are shown in the following Figs. 9-12. Fig. 9 shows the north velocity during the boost phase (ending at the instant before the opening of the engine cowl door) as a function of time since drop, which occurred at 50396.686 sec since midnight Pacific Standard Time (PST) on March 27, 2004. The plot shows the EKF-derived solution with a solid curve, the on-board navigation solution (with some prost-processing, as discussed in Ref. 58) with a dashed curve, and the GPS velocity measurement. In this plot, the IMU drift is clear, and the improvement made by using the EKF method can be seen after approximately $50 \mathrm{sec}$ after drop.

The improvement can be seen more clearly in Fig. 10, which shows the residuals between the GPS measurement and the EKF solution in the solid curve with circles at the data points, and the residuals between the GPS measurement and the on-board solution with the solid curve with squares at the data points. The dashed curves show the $+/$ - one standard deviation levels in the north velocity as output from 


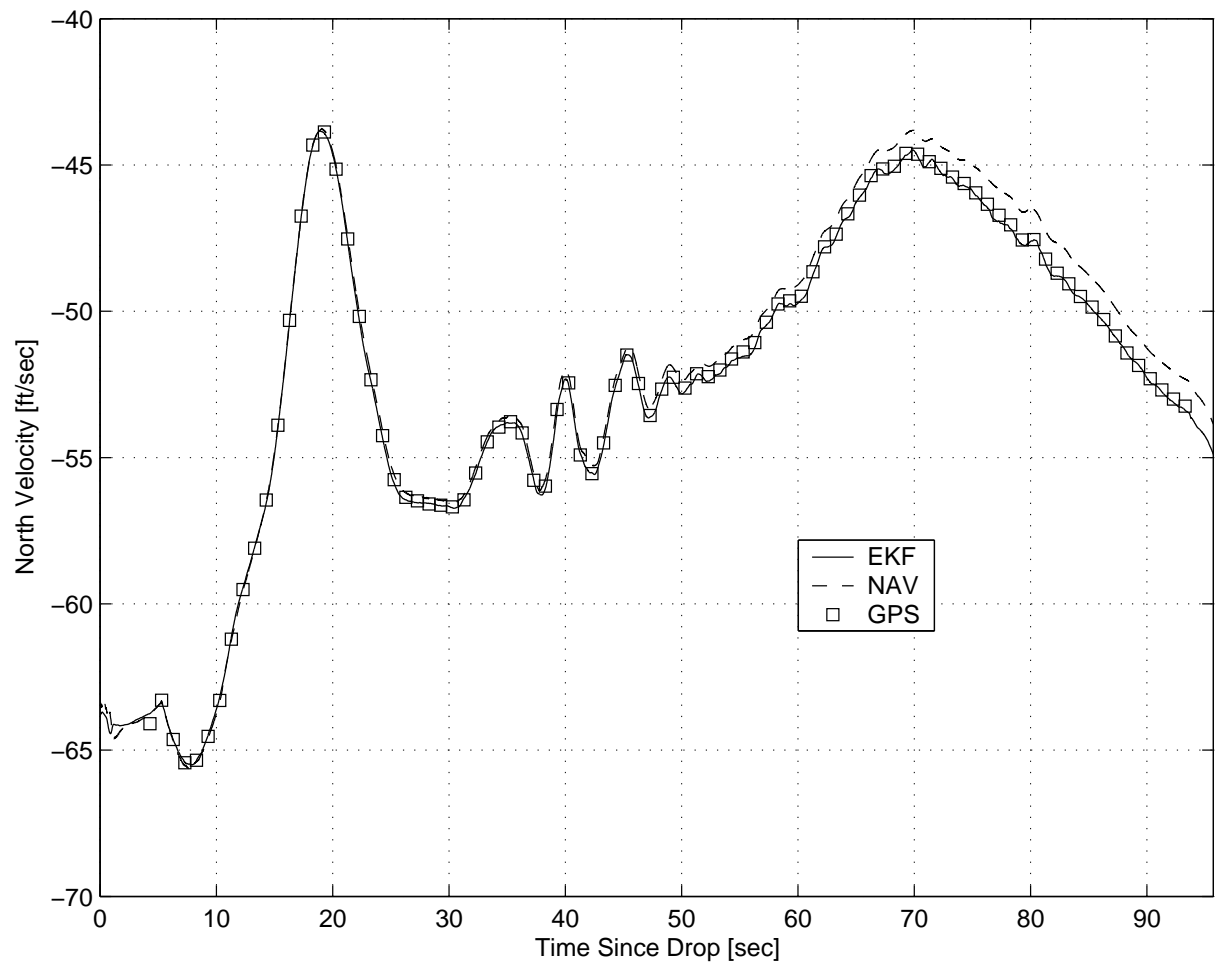

Figure 9. North Velocity Comparison During Boost

the EKF method.

Similar results occur for the other components of velocity and position measurements. The improvements are summarized in Table 3, which shows the Root Mean Square (RMS) errors between the EKF solution and the GPS solution, and the same with the on-board navigation solution. An improvement is made by the application of the EKF method in all measurement components, although some cases are more dramatic than others. The EKF-derived component to show the smallest improvement over the on-board navigation solution is the east velocity. This result is perhaps not surprising, since the east velocity measurement channel saturated during the boost, which limited the number of useful data points to be processed.

Table 3. Summary of RMS Errors

\begin{tabular}{lcccc}
\hline \hline Measurement & EKF & NAV & Ratio & Observations \\
\hline$h_{g d}$ & $21.59 \mathrm{ft}$ & $42.69 \mathrm{ft}$ & 1.977 & 571 \\
$\phi_{g d}$ & $1.202 \cdot 10^{-5} \circ$ & $3.788 \cdot 10^{-4} \circ$ & 31.51 & 571 \\
$\theta$ & $2.346 \cdot 10^{-4} \circ$ & $5.779 \cdot 10^{-4} \circ$ & 2.463 & 571 \\
$u_{g d}$ & $0.08972 \mathrm{ft} / \mathrm{s}$ & $0.3517 \mathrm{ft} / \mathrm{s}$ & 3.920 & 571 \\
$v_{g d}$ & $0.9515 \mathrm{ft} / \mathrm{s}$ & $0.9746 \mathrm{ft} / \mathrm{s}$ & 1.024 & 164 \\
$w_{g d}$ & $0.3189 \mathrm{ft} / \mathrm{s}$ & $0.5893 \mathrm{ft} / \mathrm{s}$ & 1.848 & 571 \\
\hline \hline
\end{tabular}

Finally, the time histories of the atmosphere-relative Mach number, angle of attack, and dynamic pressure are shown in Fig. 11 for the boost portion of the flight and Fig. 12 for the descent phase of the flight, which began at the instant after the engine cowl door closed. Data from the engine test phase itself is sensitive and cannot be shown. The various parameter identification maneuvers during the descent can be clearly seen in the angle of attack time history. The plots show the estimates for these quantities with a solid curve, and the $+/-3$ standard deviation values in the dashed curves. It should be noted that these uncertainties are coupled in terms of the altitude uncertainty, and therefore the estimated Mach number uncertainty, 


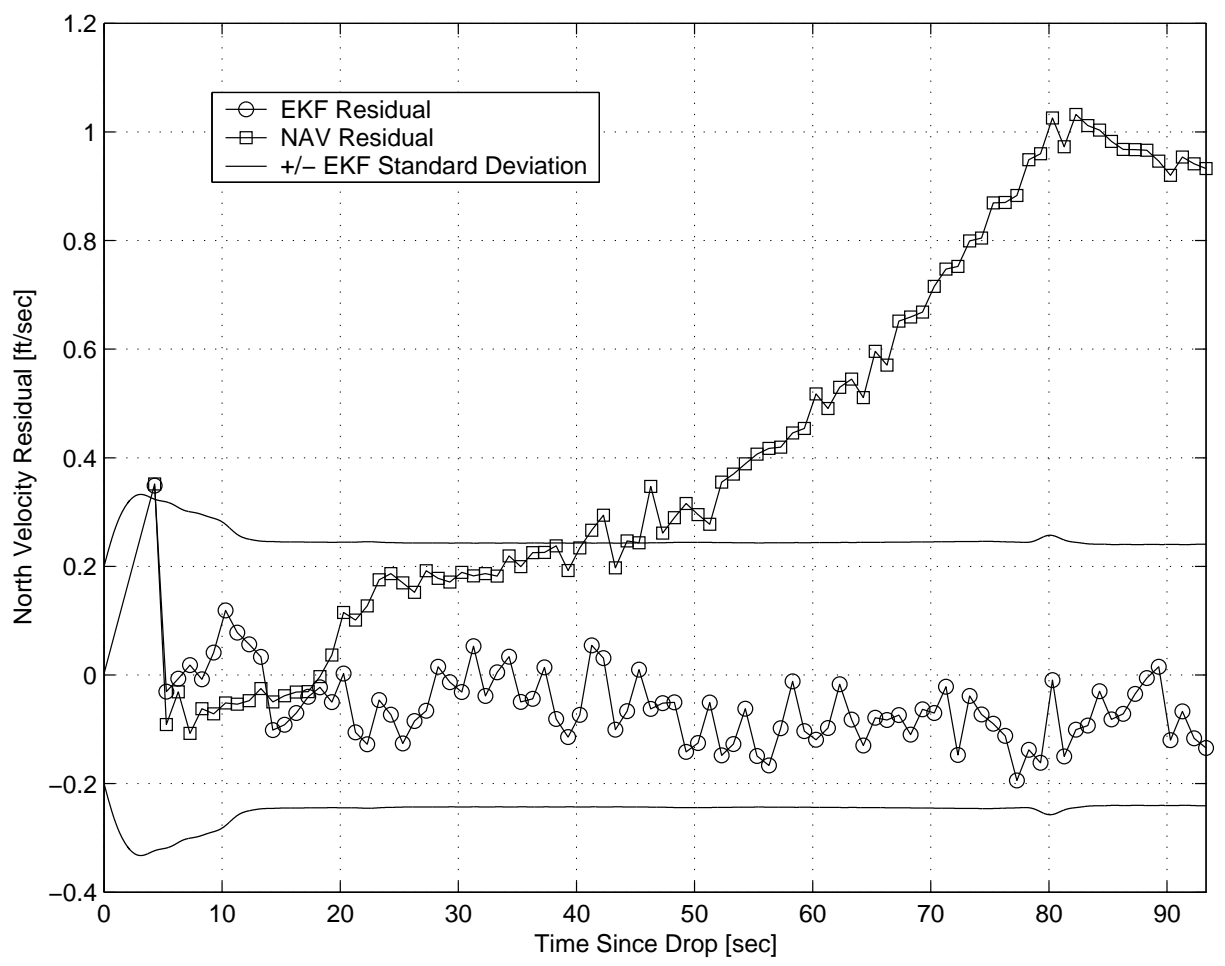

Figure 10. North Velocity Residual During Boost

for example, includes the planet-relative velocity uncertainty, wind uncertainty for a specific altitude, wind uncertainty due to altitude uncertainty, speed of sound uncertainty for the specific altitude, and speed of sound uncertainty due to uncertainty in the altitude. All of these factors were taken into consideration when computing the standard deviations of the trajectory.

\section{Conclusions}

This paper has discussed the formulation and implementation of an Extended Kalman Filtering algorithm for use in reconstructing the flight path of the Hyper-X/X-43A vehicle, using in-flight inertial measurements in the dynamic model combined with redundant measurements of the vehicle state from independent sources, particularly from the Global Positioning System. The equations of motion were formulated in a local, noninertial coordinate system, and included the effects of several systematic error sources. Equations relating the state to measured quantities were provided for a variety of data sources of interest to this problem. Data recorded during the Hyper-X flight 2 on March 27, 2004, were first preprocessed and then processed using the estimation methods discussed in this paper, leading to an improvement over the on-board navigation solution. Results for the boost and descent phases of the flight were shown. The trajectory estimate determined using the EKF method was chosen as the project Best Estimated Trajectory (Ref. 59), principally because the method allowed for a systematic procedure with which to consider all sources of uncertainty in the various measurements, and as a result was able to provide the uncertainty values for the trajectory itself.

\section{Acknowledgements}

The authors would like to thank Steve Derry for providing expertise in the area of frequency domain filtering and smoothing and for preprocessing the majority of the data for the descent trajectory, Dana McMinn for assistance with data preprocessing, Tim Moes for providing the atmosphere model and the tools to transform pressures between coordinates, and Bob James for assistance with the radar data. The authors would also like to thank Cathy Bahm, Dave Bose, Walt Engelund, Larry Huebner, Jp. Lien, John Martin, and Randy Voland for helpful discussion and support. Finally, the authors from AMA, Inc., acknowledge 

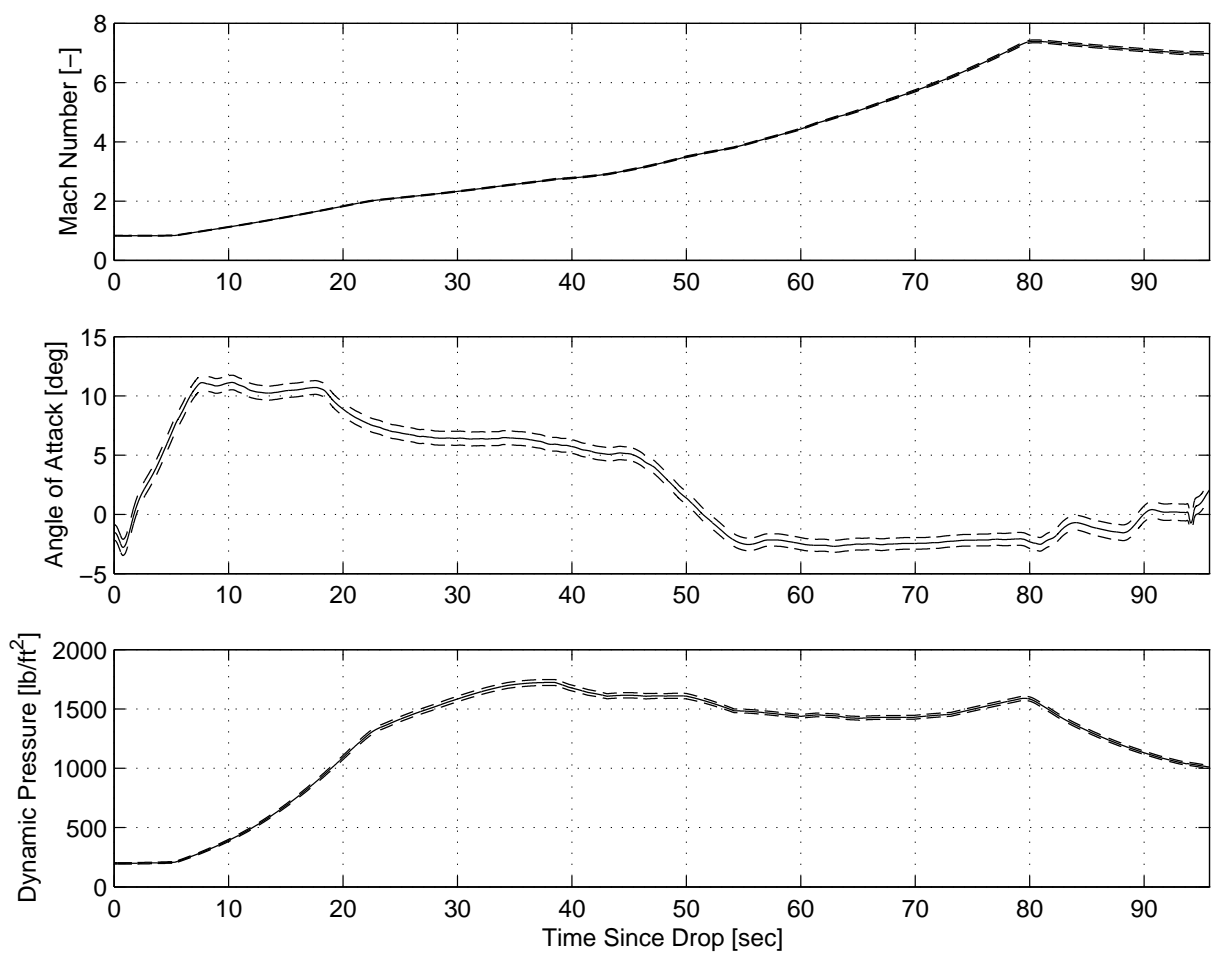

Figure 11. Mach Number, Angle of Attack, and Dynamic Pressure During Boost

funding from NASA Contracts NAS1-00135, BPA-E05204D, and BPA-NNL04AA03Z, and the authors from GWU acknowledge funding from NASA Grant NCC1-03024.

\section{References}

${ }^{1}$ McClinton, C. R., Rausch, D. R., Sitz, J., and Reukauf, P., "Hyper-X Program Status," American Institute of Aeronautics and Astronautics, AIAA Paper 2001-1910, April 2001.

${ }^{2}$ Reubush, D. E., Nguyen, L. T., Rausch, V. L., "Review of X-43A Return to Flight Activities and Current Status," American Institute of Aeronautics and Astronautics, AIAA Paper 2003-7085, December 2003.

${ }^{3}$ Engelund, W. C., Holland, S. D., Cockrell, C. E., and Bittner, R. D., "Aerodynamic Database Development for the Hyper-X Airframe-Integrated Scramjet Propulsion Experiments," Journal of Spacecraft and Rockets, Vol. 38, No. 6, 2001, pp. $803-810$.

${ }^{4}$ Woods, W. C., Holland, S. D., and DiFulvio, M., "Hyper-X Stage Separation Wind-Tunnel Test Program," Journal of Spacecraft and Rockets, Vol. 38, No. 6, 2001, pp. 811-819.

${ }^{5}$ Holland, S. D., Woods, W. C., and Engelund, W. C., "Hyper-X Research Vehicle Experimental Aerodynamics Test Program Overview," Journal of Spacecraft and Rockets, Vol. 38, No. 6, 2001, pp. 828-835.

${ }^{6}$ Huebner, L. D., Rock, K. E., Ruf, E. G., Witte, D. W., and Andrews, E. H., "Hyper-X Flight Engine Ground Testing for Flight Risk Reduction," Journal of Spacecraft and Rockets, Vol. 38, No. 6, 2001, pp. 844-852.

${ }^{7}$ Buning, P. G., Wong, T-C., Dilley, A. D., and Pao, J. L., "Computational Fluid Dynamics Predictions of Hyper-X Stage Separation Aerodynamics," Journal of Spacecraft and Rockets, Vol. 38, No. 6, 2001, pp. 820-827.

${ }^{8}$ Reubush, D. E., Martin, J. G., Robinson, J. S., Bose, D. M., and Strovers, B. K., "Hyper-X Stage Separation - Simulation Development and Results," American Institute of Aeronautics and Astronautics, AIAA Paper 2001-1802, April 2001.

${ }^{9}$ Tartabini, P. V., Bose, D. M., McMinn, J. D., Martin, J. G., and Strovers, B. K., "Hyper-X Stage Separation Trajectory Validation Studies," American Institute of Aeronautics and Astronautics, AIAA Paper 2003-5819, August 2003.

${ }^{10}$ Cockrell, C. E., Engelund, W. C., Bittner, R. D., Jentink, T. N., Dilley, A. D., and Frendi, A., "Integrated Aeropropulsive Computational Fluid Dynamics Methodology for the Hyper-X Flight Experiment," Journal of Spacecraft and Rockets, Vol. 38, No. 6, 2001, pp. 836-843.

${ }^{11}$ Cockrell, C. E., Auslender, A. H., Guy, R. W., McClinton, C. R., and Welch, S. S., "Technology Roadmap for DualMode Scramjet Propulsion to Support Space-Access Vision Vehicle Development," American Institute of Aeronautics and Astronautics, AIAA Paper 2002-5188, October 2002.

${ }^{12}$ Moses, P. L., "X-43C Plans and Status," American Institute of Aeronautics and Astronautics, AIAA Paper 2003-7084, December 2003.

${ }^{13}$ Morelli, E. A., "Flight Test of Optimal Inputs and Comparison with Conventional Inputs," Journal of Aircraft, Vol. 36, No. 2, 1999, pp. 398-397. 

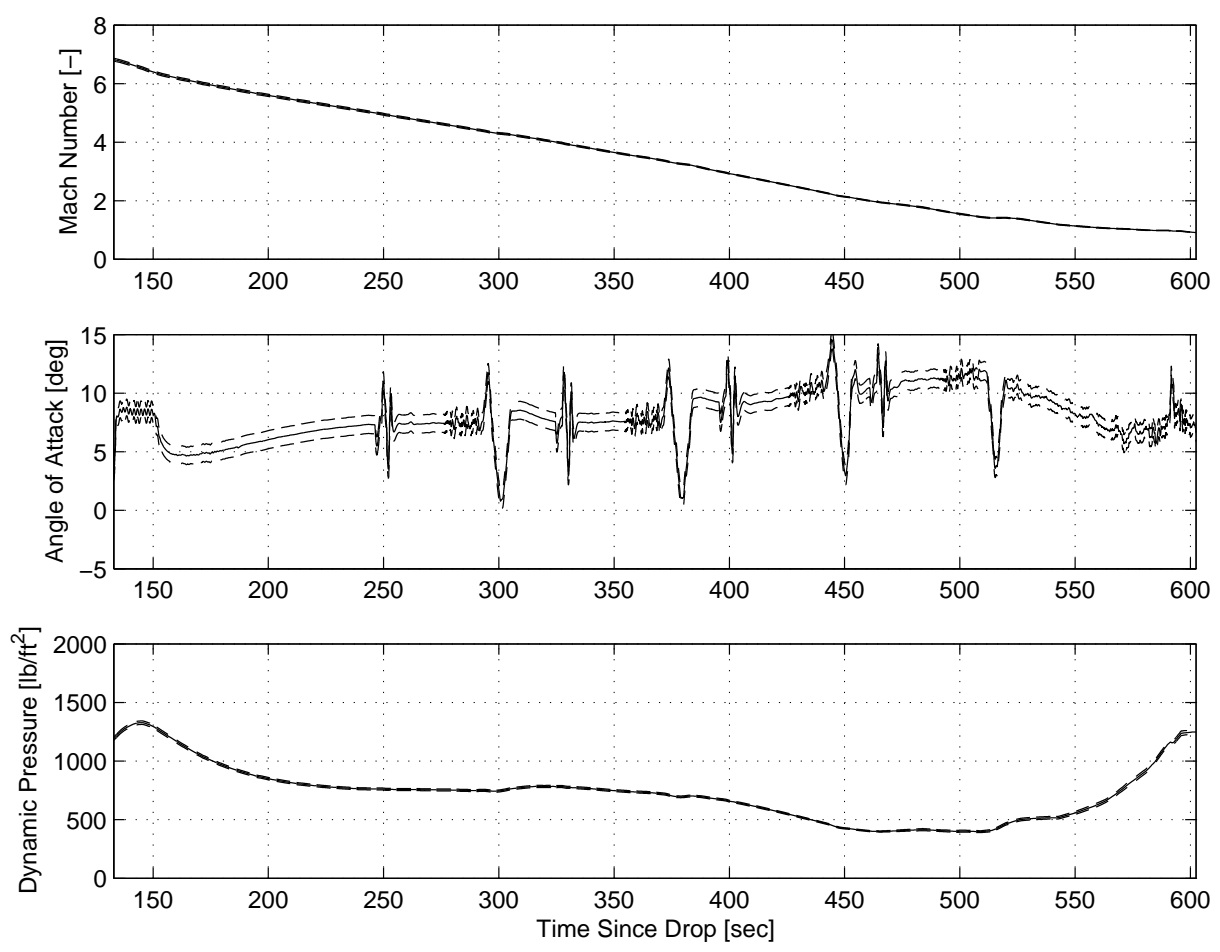

Figure 12. Mach Number, Angle of Attack, and Dynamic Pressure During Descent

${ }^{14}$ Wagner, W. E., "Re-Entry Filtering, Prediction and Smoothing," Journal of Spacecraft and Rockets, Vol. 3, No. 9, 1966, pp. 1321-1327.

${ }^{15}$ Wagner, W. E. and Serold, A. C., "Statistical Trajectory Estimation Programs: Utilization Report," NASA CR-66837, June 1969 . 1970.

${ }^{16}$ Wagner, W. E. and Serold, A. C., "Formulation on Statistical Trajectory Estimation Programs," NASA CR-1482, January

${ }^{17}$ Anon., "SV-5D PRIME: Final Flight Test Summary," Martin Marietta Engineering Report 14465, September 1967.

${ }^{18}$ Jones, J. F., "Development and Performance Analysis of a Trajectory Estimator for an Entry Through the Martian Atmosphere," American Institute of Aeronautics and Astronautics, AIAA Paper 72-953, September 1972.

${ }^{19}$ Hopper, F. W., "Trajectory, Atmosphere, and Wind Reconstruction from Viking Entry Measurements," American Astronautical Society, AAS Paper 75-068, July 1975.

${ }^{20}$ Euler, E. A., Adams, G. L., and Hopper, F. W., "Design and Reconstruction of the Viking Lander Descent Trajectories," Journal of Guidance, Control, and Dynamics, Vol. 1, No. 5, 1978, pp. 372-378.

${ }^{21}$ Sabin, M. L., "Linear Filtering of Ballistic-Entry Probe Data for Atmospheric Reconstruction," Journal of Spacecraft and Rockets, Vol. 12, No. 2, 1975, pp. 66-73.

${ }^{22}$ Compton, H. R., Blanchard, R. C., and Findlay, J. T., "Shuttle Entry Trajectory Reconstruction Using Inflight Accelerometer and Gyro Measurements," American Institute of Aeronautics and Astronautics, AIAA Paper 79-0257, January 1979.

${ }^{23}$ Compton, H., Findlay, J., Kelly, G., and Heck, M., "Shuttle (STS-1) Entry Trajectory Reconstruction," American Institute of Aeronautics and Astronautics, AIAA Paper 81-2459, November 1981.

${ }^{24}$ Karlgaard, C. D. and Bose, D. M., "Hyper-X Post-Flight Trajectory Reconstruction Tool Interim Report," Analytical Mechanics Associates, Inc., AMA Report 02-41, December 2002.

${ }^{25}$ Jaswinski, A. H., Stochastic Processes and Filtering Theory, Academic Press, San Deigo, CA, 1970.

${ }^{26}$ Gelb, A. (Ed.), Applied Optimal Estimation, The M.I.T. Press, Cambridge, MA, 1974.

${ }^{27}$ Stengel, R. F., Optimal Control and Estimation, Dover Publications, Inc., New York, NY, 1994.

${ }^{28}$ Zarchan, P. and Musoff, H., Fundamentals of Kalman Filtering: A Practical Approach, AIAA Progress in Astronautics and Aeronautics, Vol. 190, 2000.

${ }^{29}$ Fraser, D. C. and Potter, J. E., "The Optimum Linear Smoother as a Combination of of Two Optimum Linear Filters," IEEE Transactions on Automatic Control, Vol. 14, No. 8, 1969, pp. 387-390.

${ }^{30}$ Spencer, D. A., Blanchard, R. C., Braun, R. D., Kallemeyn, P. H., and Thurman, S. W., "Mars Pathfinder Entry, Descent, and Landing Reconstruction," Journal of Spacecraft and Rockets, Vol. 36, No. 3, 1999, pp. 357-366.

${ }^{31}$ Rao, A. V., "Minimum-Variance Estimation of Reentry Debris Trajectories," Journal of Spacecraft and Rockets, Vol. 37, No. 3, 2000, pp. 366-373.

${ }^{32}$ Tardy, J. M. and Kluever, C. A., "Estimation and Prediction of Orbital Debris Reentry Trajectories," Journal of Spacecraft and Rockets, Vol. 39, No. 6, 2002, pp. 845-851. 
${ }^{33}$ Thirkettle, A., Steinkopf, M., and Joeseph-Gabriel, E., "The Mission and Post-flight Analysis of the Atmospheric Reentry Demonstrator," European Space Agency Bulletin, Vol. 109, No. 2, 2002, pp. 56-63.

${ }^{34}$ Withers, P., Towner, M. C., Hathi, B., and Zarnecki, J. C., "Analysis of Entry Accelerometer Data: A Case Study of Mars Pathfinder," Planetary and Space Science, Vol. 51, No. 9/10, 2003, pp. 541-561.

${ }^{35}$ Vallado, D. A., Fundamentals of Astrodynamics and Applications, McGraw-Hill, New York, NY, 1997.

${ }^{36}$ Sofair I., "Improved Method for Calculating Exact Geodetic Latitude and Altitude," Journal of Guidance, Control, and Dynamics, Vol. 20, No. 4, 1997, pp. 824-826.

${ }^{37}$ Sofair, I., "Improved Method for Calculating Exact Geodetic Latitude and Altitude Revisited," Journal of Guidance, Control, and Dynamics, Vol. 23, No. 2, 2000, pp. 369.

${ }^{38}$ Nievergelt, Y. and Keeler, S. P., "Computing Geodetic Coordinates in Space," Journal of Spacecraft and Rockets, Vol. 37, No. 2, 2000, pp. 293-296.

${ }^{39}$ Anon., "WGS-84 Implementation Manual," Version 2.4, February 1998.

${ }^{40}$ Phillips, W. F., Hailey, C. E., and Gebert, G. A., "Review of Attitude Representations Used for Aircraft Kinematics," Journal of Aircraft, Vol. 38, No. 4, 2001, pp. 718-737.

${ }^{41}$ Heck, M. L., Findlay, J. T., Kelly, G. M., and Compton, H. R., "Adaptation of a Strapdown Formulation for Processing Inertial Platform Data," Journal of Guidance, Control, and Dynamics, Vol. 7, No. 1, 1984, pp. 15-19.

${ }^{42}$ Bose, D. M. and Karlgaard, C. D., "Detailed Trajectory Reconstruction Using STEP," Analytical Mechanics Associates, Inc., AMA Report 01-31, September 2001.

${ }^{43}$ Shuster, M. D., "Constraint in Attitude Estimation Part I: Constrained Estimation," The Journal of the Astronautical Sciences, Vol. 51, No. 1, 2003, pp. 51-74.

${ }^{44}$ Shuster, M. D., "Constraint in Attitude Estimation Part II: Unconstrained Estimation," The Journal of the Astronautical Sciences, Vol. 51, No. 1, 2003, pp. 75-101.

${ }^{45}$ Markley, F. L., "Attitude Error Representations for Kalman Filtering," Journal of Guidance, Control, and Dynamics, Vol. 26, No. 2, 2003, pp. 311-317.

${ }^{46}$ Pittelkau, M. E., "An Analysis of the Quaternion Attitude Determination Filter," The Journal of the Astronautical Sciences, Vol. 51, No. 1, 2003, pp. 103-120.

${ }^{47}$ Farrell, J. A. and Barth, M., The Global Positioning System and Inertial Navigation: Theory and Practice, McGraw-Hill, New York, NY, 1999.

${ }^{48}$ Rogers, R. M., Applied Mathematics in Integrated Navigation Systems, American Institute of Aeronautics and Astronautics, Reston, VA, 2000.

${ }^{49}$ Toniolo, M. D., "Flight Data Preprocessing Tool (PREPR) User's Guide," Analytical Mechanics Associates, Inc., AMA Report 04-16, July 2004.

${ }^{50}$ Morelli, E. A., "Estimating Noise Characteristics from Flight Test Data Using Optimal Fourier Smoothing," Journal of Aircraft, Vol. 32, No. 4, 1995, pp. 689-695.

${ }^{51}$ Morelli, E. A., "System Identification Programs for Aircraft (SIDPAC)," American Institute of Aeronautics and Astronautics, AIAA Paper 2002-4702, August 2002.

${ }^{52}$ Anon., "H-764 System Description," Honeywell, Inc., Report 1096-1339, February 1997.

${ }^{53}$ Lecheler, B. A., "Math Model, Flight Management Unit, Hyper-X," Honeywell, Inc., Report ED 23418, October 1997.

${ }^{54}$ Anon., "Hyper-X Flight Management Unit System Performance During Flight Profile Results," Honeywell, Inc., July 1998.

55 James, B., "Analysis of Radar Tracking Data: Hyper-X Mission 3-27-2004," Hyper-X Project Report, April 252004.

${ }^{56}$ Besada Portes, J. A., Herrero, J. G., and de Miguel Vela, G., "Radar Bias Correction Based on GPS Measurements for ATC Applications," IEE Proceedings of Radar, Sonar, and Navigation, Vol. 149, No. 3, 2002, pp. 137-144.

${ }^{57}$ Anon., "X-43A Flight \#2 Reference Atmosphere," NASA Dryden Flight Research Center Hyper-X Report HX-DFRC0368 Rev. B, April 23, 2004.

${ }^{58}$ Anon., "X-43A Flight \#2 Best Estimated Trajectory, " NASA Dryden Flight Research Center Hyper-X Report HXDFRC-0371, April 27, 2004.

${ }^{59}$ Anon., "X-43A Flight 2 Official Best Estimated Trajectory," NASA Dryden Flight Research Center Hyper-X Report HX-DFRC-0389, July 21, 2004. 